\title{
Pharmacocinétique / pharmacodynamie clinique des antibiotiques
}

Chapitre 35 du "Précis de Bactériologie clinique", J. Freney, F. Renaud, W. Hansen, C. Bollet éditeurs, $3^{\text {ème }}$ édition, Éditions Eska, Paris et Alexandre Lacassagne, Lyon, 2000, pp. 715-731 http://www.eska.fr

\author{
Pascal Maire, Stéphane Corvaisier, Michel Bouvier d'Yvoire, Daniel Claude, Xavier \\ Barbaut, Gérard Carret, François Jehl, Jean-Christophe Thalabard, Roger W. Jelliffe
}

ADCAPT - Service Pharmaceutique, Hôpital Antoine Charial, Hospices Civils de Lyon, 40, avenue de la Table de Pierre, 69340 Francheville; et Laboratory of Applied Pharmacokinetics, School of Medicine, University of Southern California, Los Angeles, CA.

\section{INTRODUCTION}

Même si nous essayons de l'oublier, l'expérience clinique quotidienne nous rappelle constamment que, soumis à la thérapeutique anti-infectieuse, les patients diffèrent dans leurs réponses cliniques. Pour une grande majorité de molécules récemment développées par l'industrie pharmaceutique et finalement sélectionnées par le processus réglementaire, nous l'oublions car les différences dans les réponses sont minimes, ou bien l'index thérapeutique est grand, de sorte que la variabilité est sans importance. Cependant, pour des traitements qui risquent d'être toxiques ou au contraire inefficaces, il ne nous est plus possible d'ignorer cette variabilité (46) qui peut désormais être maîtrisée si nécessaire (18).

Cette variabilité est responsable de divergences entre ce qu'espère le clinicien, au début $\mathrm{du}$ traitement, en terme de cible (concentration en médicament, effet pharmacologique, attracteur d'un système dynamique) atteignable par un schéma d'administration spécifique et ce qu'il observera réellement par la suite. Dans ces conditions, ne vaudrait-il pas mieux prédire ces divergences - dans le futur - plutôt que les constater - au présent - ? Et mieux encore prédire pour corriger, c'est-à-dire contrôler?

Une partie de la pharmacologie, la pharmacocinétique, étudie le sort, le devenir, des médicaments introduits dans l'organisme, de l'absorption à l'élimination en passant par la distribution. Une autre partie, la pharmacodynamie, a pour objet l'étude de l'action exercée par les médicaments sur l'organisme. Selon une autre formulation, la pharmacocinétique établit un rapport entre les posologies administrées, les concentrations en médicament obtenues et leur variation dans le temps alors que la pharmacodynamie relie ces concentrations aux réponses pharmacologiques qui peuvent être des critères intermédiaires (par exemple normalisation de la température corporelle, normoleucocytose ou encore stérilité des cultures bactériennes) ou finaux d'efficacité (par exemple mortalité au dixième jour, sans aller jusqu'à une définition de la guérison) ou de toxicité (par exemple toxicité tubulaire et retentissement glomérulaire). 
La pharmacodynamie des anti-infectieux (antibactériens, antifongiques et antiviraux) a pour cible thérapeutique le microorganisme et ses métabolismes, cible qui ne fait pas partie stricto sensu de l'organisme, même malade. Elle n'est donc pas dans le prolongement direct de la phase pharmacocinétique proprement humaine (même s'il existe une phase pharmacocinétique bactérienne pour partie responsable peut-être de certains effets postantibiotiques).

En complément de la pharmacocinétique expérimentale qui a pour objectif de définir les grandes caractéristiques des anti-infectieux, leur "carte d'identité", principalement en vue de leur enregistrement réglementaire, la pharmacocinétique clinique - appliquée - a pour objectifs de décrire, d'estimer (pharmacocinétique de population) pour contrôler (contrôle adaptatif optimal des posologies) la variabilité des thérapeutiques médicamenteuses, entre individus d'une même population et de proposer des schémas d'administration des médicaments.

Dans les deux premières éditions du Manuel de bactériologie clinique : tout d'abord nous avions insisté et essentiellement sur l'intérêt de prédire les concentrations plasmatiques des antibiotiques qui présentaient des risques d'accumulation et de toxicité (essentiellement les aminosides et glycopeptides selon les schémas posologiques de la période considérée), ensuite nous avions procédé à une revue des techniques de pharmacocinétique de population $(32,35)$, et enfin nous avions présenté les premières approches de pharmacodynamie des antiinfectieux (35).

Depuis la seconde édition, d'autres modalités d'administration des aminosides et glycopeptides ont, semble-t-il, repoussé au second plan les risques d'accumulation et de toxicité. En revanche, l'intérêt pour un monitoring de l'efficacité et de la pharmacodynamie des anti-infectieux s'est encore accru, notamment pour des molécules de toxicité aiguë faible, de diffusion extraplasmatique importante, d'action intracellulaire (par exemple fluoroquinolones, $\beta$-lactamines, macrolides,...). Malheureusement pour ces produits, d'une part, il n'existe pas encore de kits de dosages automatisés permettant leur monitoring à grande échelle, d'autre part, les données utilisables comme cibles pour les nouvelles techniques d'adaptation de posologie sont encore peu nombreuses ; il s'agit toujours actuellement presque exclusivement de concentrations sériques.

Dans ces conditions, il nous a semblé judicieux, pour cette nouvelle édition du Précis de bactériologie clinique, d'abandonner les références à des techniques de contrôle de concentrations plasmatiques qui, certes, ont prouvé leur efficacité(26), sont encore très utilisées en pratique clinique courante mais qui sont progressivement moins sollicitées (nous en développerons certaines raisons par la suite) ; et, à la place de développer : (i) les grands principes de l'adaptation de posologie des anti-infectieux qui sont à la base des techniques modernes désormais disponibles afin de préparer l'infectiologue aux bases théoriques des techniques futures, (ii) une revue générale des indices déjà assez largement utilisés et des modèles pharmacodynamiques d'action des antibiotiques susceptibles d'être utilisés dans un futur proche par les modélisations pharmacocinétique / pharmacodynamique cliniques d'action des antibiotiques. Cependant, dans une troisième et dernière partie, (iii) nous avons continué d'indiquer les attitudes pratiques de pharmacocinétique clinique, désormais consacrées par l'usage, qui permettent, à moindre coût, d'intégrer certains des aspects pharmacodynamiques les plus récents. 


\section{BASES THEORIQUES DE L'ADAPTATION DE POSOLOGIE EN ANTIBIOTHERAPIE}

\subsection{MODELES ET CONTROLE DE SYSTEMES}

La théorie de l'adaptation de posologie se réfère au contrôle de systèmes. Un système de contrôle est lui-même un ensemble de composants connectés de manière à commander, diriger ou réguler lui-même ou un autre système. Un exemple simple est celui d'un thermostat pilotant un grill pain électrique : l'intensité parcourant la résistance (une entrée) est adaptée, selon un modèle, par le thermostat en fonction de la température qu'il mesure (une sortie) et des objectifs qu'on lui a définis (une cible qui est dans cet exemple une température à atteindre et à maintenir pendant un temps donné). Un modèle ne reproduit que certains aspects du système considéré, de la réalité qui nous intéresse : ce n'est jamais une représentation exacte du processus réel, laquelle ne pourrait être tentée qu'au risque de perdre les avantages du modèle par rapport au système réel. En effet, le modèle doit être construit en fonction des objectifs poursuivis et des éléments majeurs qui permettent de les réaliser. Ainsi, en ce qui nous concerne, le contrôle de la thérapeutique qui est l'objectif fixé. Il est inutile, voire nuisible, de compliquer ce modèle plus que nécessaire; d'autant plus que, pour l'imaginer comme pour l'utiliser par la suite, il faut disposer de suffisamment d'informations. De la richesse des connaissances disponibles, en général (paramètres, descripteurs physiologiques ou covariables, mécanismes antibactériens...) et pour chaque cas particulier (mesures réalisées anthropométriques, biologiques, pharmacologiques...), dépend le succès de la modélisation et par-là même de l'adaptation de posologie. Les connaissances devront permettre d'établir la structure du modèle, d'avoir une idée des valeurs possibles de ses paramètres et de définir les conditions initiales avant toute simulation. Les conditions initiales et les paramètres du modèle peuvent être fixés initialement mais doivent être réévalués périodiquement.

En pharmacocinétique clinique, un modèle mathématique décrivant le comportement du médicament dans l'organisme du patient, est utilisé pour simuler et donc prédire, parallèlement à l'administration réelle du médicament, des réponses à des entrées numériques. Dans le cas d'une représentation compartimentale, le modèle mathématique peut être représenté par un ensemble d'équations différentielles, de conditions initiales et de limites (issues de la physique ou de la physiologie). Dans l'une au moins de ces équations est incorporé un vecteur d'entrées correspondant aux doses successivement administrées ; toutes peuvent comporter un terme correspondant aux différents bruits du système. L'état du système, à un instant donné, est un résumé de toute l'information disponible sur son passé susceptible d'influencer son futur (33).

Les premières tentatives de modélisation et de contrôle des systèmes anti-infectieux / hôte / microorganisme ont été exclusivement pharmacocinétiques. En effet les premières versions des logiciels d'adaptation de posologie (USC*Pack, PKS,...) utilisées en situation clinique n'utilisaient que des modèles pharmacocinétiques : compartimentaux linéaires, à un ou deux compartiments, avec ou sans compartiment d'absorption (34).

Ces modèles étaient remarquablement bien adaptés pour décrire et contrôler le comportement pharmacocinétique des aminosides et des glycopeptides. Avec eux, il était possible de prédire les concentrations sanguines (centrales) et éventuellement périphériques 
(simulées dans un compartiment périphérique virtuel) en médicament après injection intraveineuse, intramusculaire, voire administration orale pour d'autres que les produits cités ci-dessus $(26,34)$; un exemple graphique d'un tel suivi thérapeutique en terme de concentrations plasmatiques est présenté dans la dernière partie de ce chapitre.

Mais à partir de ces prédictions, il restait nécessaire d'extrapoler en termes de toxicité, d'efficacité ou tout simplement de diffusion sur les lieux d'action de l'antibiotique. C'est la raison pour laquelle nous avons envisagé, dans certaines infections, de compléter ce modèle pharmacocinétique d'un modèle physio-pathologique sous la forme d'un compartiment de diffusion connectable, selon nécessités au compartiment central ou au compartiment périphérique (31).

Le premier modèle de diffusion radiale a été développé pour simuler les concentrations à différents niveaux d'une végétation d'endocardite infectieuse (6). Les traitements de l'endocardite nécessitent généralement une antibiothérapie prolongée. L'individualisation des régimes posologiques a rendu possible de telles thérapeutiques même chez des patients présentant une fonction rénale très altérée (34). De tels modèles physiopathologiques de diffusion ont montré (6) le bien-fondé des règles empiriques de traitement de l'endocardite infectieuse : nécessité d'un traitement à la fois le plus précoce possible, suffisamment prolongé et surtout à des doses conséquentes pour permettre des concentrations actives au coeur de la végétation, mettant à profit la notion de concentration-dépendance des aminosides (cette notion sera discutée plus loin). Cependant le problème de l'efficacité - de la pharmacodynamie - demeure : cette modélisation physiopathologique nécessite toujours d'extrapoler en termes d'efficacité à partir de valeurs de concentrations en antibiotiques. C'est la raison pour laquelle il est désormais possible de raccorder à ces modèles pharmacocinétique / physiopathologique des modèles pharmacodynamiques d'action des antibiotiques (36).

Le modèle de Hill est largement utilisé en pharmacologie pour traiter des données expérimentales in vitro (organes isolés) ou in vivo (animales). Zhi l'a proposé pour représenter l'antagonisme entre la croissance bactérienne et la bactéricidie (55); Li l'a complexifié (29). Les comparaisons entre ce modèle pharmacodynamique de bactéricidie et les indices de bactéricidie généralement en usage, dits "paramètres pharmacodynamiques", ont été faites (7). Il est cependant nécessaire de préciser dans quelles conditions de tels modèles permettent de prédire ou de simuler l'évolution clinique.

Remarquons déjà que les modèles précédents ne sont adaptés qu'à des antibiotiques pas ou très faiblement liés aux protéines plasmatiques et ne prennent pas non plus en compte l'éventuelle fixation sur des sites d'action ou lieux de perte. Il est possible cependant de gérer la fixation protéique en ajoutant à ces modèles un compartiment virtuel (donc une équation différentielle modifiant les précédentes) représentant cette captation réversible. Quel que soit le compartiment du lieu d'action, seule la fraction libre est active.

\subsection{THERAPEUTIQUE ANTIBIOTIQUE : DETERMINISTE OU ALEATOIRE ?}

Lorsque le déterminisme - conception mécanique classique newtonnienne - s'applique à un système, "nous devons [pouvons] envisager l'état présent de l'univers [celui-ci] comme l'effet de son état antérieur, et comme la cause de celui qui va suivre" (P.S. Laplace, 1825), ce qui nécessite (i).de connaître la loi, le modèle, qui peut le représenter; (ii).avec une précision 
adéquate d'être capable de faire des observations sur le comportement du système ; (iii).de s'assurer de relations linéaires entre entrées et sorties par rapport aux paramètres du modèle, dans un environnement pas trop bruité (34). Les conditions précédentes n'excluent malgré tout pas le risque d'un chaos déterministe.

Lorsque les conditions précédentes ne sont pas réunies, le comportement du modèle peut être considéré comme stochastique, aléatoire, et dans cette conjoncture l'état du système à un instant $(\mathrm{n})$ ne peut être décrit qu'en fonction de l'état immédiatement antérieur (n-1), comme dans le cas du mouvement brownien, il s'agit alors d'un processus markovien.

Les modèles pharmacocinétiques simples (compartimentaux, d'ordre 1, sans mécanisme saturable, linéaires) permettent d'être déterministe, et à partir de la reconstruction du passé (à condition qu'au début de la thérapeutique - d'application de la commande - à (t0) les concentrations en médicament soient nulles), de prédire les concentrations futures, sériques ou périphériques, en médicament, théoriquement d'un temps $(\mathrm{t} 0)$ à $(\mathrm{t}+\propto)$. En plus des premières mesures de concentrations en médicament, ils peuvent utiliser une information initiale (des valeurs initiales pour les paramètres), sous forme de probabilités a priori actualisée périodiquement par la suite.

Dès qu'il est nécessaire de recourir à des modèles plus complexes (d'ordre 0 , avec des mécanismes saturables, pharmacocinétiques / pharmacodynamiques en particulier, non linéaires), l'approche déterministe n'est plus possible, notamment en raison de non linéarités introduisant la possibilité d'attracteurs très différents en fonction d'une légère variation des paramètres estimés (sensibilité aux conditions initiales). D'autre part, les bruits et perturbations constituant des entrées aléatoires, un système pharmacocinétique n'est déterministe qu'en première approximation, donc stochastique par souci de généralité. Il est alors nécessaire d'utiliser des approches stochastiques qui fournissent des probabilités de trajectoires horizon par horizon; un horizon (au sens d'une perspective sur l'avenir) étant un intervalle de temps incluant des événements (doses administrées, mesures de concentrations, changement de variables cliniques,...). Ainsi, dans un horizon (n), les probabilités postérieures $\mathrm{du}$ précédent (n-1) sont utilisées comme informations a priori et actualisées (donc $a$ posteriori) pour devenir les a priori du suivant $(\mathrm{n}+1)$.

En fait, même sans recourir aux techniques récentes d'adaptation de posologie, ces concepts sont déjà couramment utilisés par l'infectiologue.

\subsection{POSOLOGIE FIXÉE $A$ PRIORI}

Dans la pratique médicale actuelle, l'utilisation d'une posologie fixe, quelle que soit la situation clinique, résulte de l'assimilation de tous les patients traités à un modèle pharmacocinétique standard dont les paramètres, au vu des études cliniques pré-A.M.M., ont été fixés à une valeur de référence (technique 1) : par exemple, roxythromycine $150 \mathrm{mg}, 2$ fois par jour, le souhait de l'industriel étant de montrer par les études de pharmacocinétique expérimentale qu'il est possible "d'oublier" par la suite en clinique l'étape pharmacocinétique et de donner une posologie standard pour une même réponse de la totalité de l'espèce humaine; c'est possible lorsque la zone thérapeutique est large et la variabilité faible ou sans conséquence sur les phases pharmacocinétique et pharmacodynamique. 


\subsection{POSOLOGIE ADAPTÉE PAR DES COVARIABLES}

Quand ces posologies standard sont adaptées en fonction de descripteurs cliniques, comme par exemple : le poids, l'âge ou la clairance de la créatinine, une information supplémentaire personnalise déjà les paramètres pharmacocinétiques fixés initialement. Il a été montré au préalable que ces descripteurs cliniques, ou covariables, sont des variables corrélées aux paramètres pharmacocinétiques au sein de la population traitée et qu'il est nécessaire d'en tenir compte pour éviter le sous-dosage ou l'accumulation. Par exemple, il a été démontré, au niveau populationnel, qu'il existait une corrélation entre la constante d'élimination totale (Kel) des aminosides et la clairance de la créatinine $(\mathrm{CCr})$ :

$$
\mathrm{Kel}=\mathrm{Ks} * \mathrm{CCr}+\mathrm{Ki}
$$

$\mathrm{Ki}$ représentant l'élimination non rénale et Ks la pente d'élimination rénale (34). L'utilisation intuitive de ces régressions linéaires pour modifier les posologies se fait souvent par l'intermédiaire de règles de trois, de réglettes ou d'abaques. De telles techniques ne sont plus utilisables lorsque plusieurs covariables affectent simultanément plusieurs paramètres pharmacocinétiques (par exemple le poids et la clairance de la créatinine modifiant simultanément et respectivement le volume de distribution et la constante d'élimination de la vancomycine). Il est alors plus simple de recourir à une simulation avec les paramètres initiaux modifiés simultanément par les covariables (technique 2).

\subsection{ESTIMATION INDIVIDUELLE DES PARAMÈTRES PAR RÉGRESSION}

Pour un certain nombre de médicaments, une marge thérapeutique étroite est associée à une importante variabilité des paramètres pharmacocinétiques sans être explicable par des covariables aisément accessibles (par exemple le phénotype). Il est alors nécessaire de recourir à des mesures de concentrations plasmatiques pour estimer les paramètres pharmacocinétiques individuels par des techniques de régression, représentées à l'origine par celles de régression linéaire sur papier semi-logarithmique. Les techniques de régression non linéaire évitent désormais cette transformation logarithmique qui introduisait, par anamorphose, un modèle supplémentaire et ne permettait pas une gestion correcte de l'erreur associée à la mesure ; elles permettent d'estimer les paramètres pharmacocinétiques d'un médicament en situation clinique avec administrations répétées et mesure des concentrations plasmatiques après différentes doses, tout cela sans avoir besoin d'attendre l'équilibre (terminologie pharmacocinétique) ou plus exactement en termes d'automatique le régime permanent. Elles font appel à différentes procédures d'optimisation, notamment à celles dites directes, comme celle du simplexe selon Nelder et Mead. Les techniques de régression non linéaire pondérées associent de plus une crédibilité (information de Fisher) à chaque mesure (technique 3). Malheureusement ces techniques, face à un nouveau cas, ne prennent pas en compte l'expérience antérieurement acquise sur ce type de patient (elles forcent l'infectiologue à "oublier" son expérience passée) et nécessitent un nombre important de prélèvements, même pour des modèles assez simples (34).

\subsection{ESTIMATION "BAYÉSIENNE"}

Les techniques d'estimation dites "Bayésiennes" pallient les inconvénients de chacune des techniques précédentes en les associant. Une information a priori sur la population est d'emblée utilisée (technique 1), associée à l'incorporation de covariables (technique 2) et aux 
résultats des dosages sanguins de médicaments (technique 3). Elles sont utilisables dès la première mesure de concentration, sans qu'il soit nécessaire d'attendre le régime permanent (34). Elles sont dites Bayésiennes car elles font appel aux probabilités conditionnelles : à une information initiale a priori sur les paramètres au sein de la population de référence sous la forme d'une densité de probabilité jointive gaussienne, elles substituent, au fur et à mesure des estimations, une information actualisée $a$ posteriori.

A l'absence totale d'information face à un nouveau patient, le thérapeute va préférer utiliser une information, même s'il la sait imparfaite, partiellement adaptée ou incomplète. Dès que possible, il actualisera cette information et la personnalisera, en procédant à des mesures, même s'il les sait entachées d'erreurs et d'imprécisions. L'algorithme va alors combiner ces informations en les pondérant de leurs incertitudes respectives ; n'est-ce pas ainsi que le clinicien procède dans sa pratique quotidienne et même en dehors de sa profession, dans la vie de tous les jours?

Différentes techniques auxiliaires, la D-optimalité par exemple, permettent de proposer les temps de prélèvements futurs où l'information sur l'état du système sera maximale. Mais les estimateurs Bayésiens des paramètres (technique 4), s'ils gèrent les erreurs associées aux mesures des concentrations, ne prennent pas en compte les autres sources d'incertitudes : erreur sur le modèle, dite structurale, erreurs sur les temps de prélèvement et d'administration ou enfin erreur sur les doses administrées. Essayer d'incorporer toutes ces erreurs en "gonflant" la part de l'erreur analytique qui pondère les dosages plasmatiques, est un pis-aller qui s'éloigne de la réalité. Enfin, ces estimateurs calculent les paramètres de modèles déterministes, comme précédemment défini, toujours linéaires.

\subsection{ESTIMER DES PARAMÈTRES POUR CONTRÔLER UN SYSTÈME}

A partir de l'estimation des paramètres, en vertu du principe de séparation estimation / contrôle $^{1}$, il est possible, dans un second temps, de contrôler le système et de faire par simulation des prévisions en fonction des cibles que souhaite atteindre le clinicien et donc de définir la thérapeutique future. Cela est réalisé par la deuxième partie du programme, le contrôleur, qui nécessite une intervention humaine. Il s'agit notamment d'exprimer les cibles à atteindre simultanément en terme d'efficacité et de toxicité (25) : en fonction de la gravité de l'infection, quelle est la concentration plasmatique, périphérique ou tissulaire, qu'il souhaite atteindre, à quel moment et pendant combien de temps ? De même, en fonction du risque vital encouru par le patient, quelle est la concentration qu'il n'accepte pas de dépasser en terme de toxicité et à quel moment? Plus généralement, que veut-on contrôler : des concentrations, des intervalles de temps entre concentrations maxima et minima, la dose totale administrée ou encore le rythme d'administration lui-même?

Les paramètres estimés sont utilisés pour contrôler le système dans le futur et atteindre les cibles fixées. Dans un premier temps, l'intervalle posologique le plus adapté est calculé puis corrigé en fonction des possibilités de réalisation pratique; dans un deuxième, les doses théoriques à administrer sont modifiées de la même manière ; enfin sont déterminés les moments optimaux pour de futurs prélèvements où l'information collectée sera maximale $(26,34)$.

\footnotetext{
${ }^{1}$ Cette solution, bien que sous-optimale, est souvent acceptée en automatique.
} 


\subsection{CONTRÔLEURS À MODÈLES MULTIPLES}

Ces contrôleurs, également Bayésiens car ils actualisent des probabilités initiales face à un nouveau patient, sélectionnent dans une banque de modèles les jeux de paramètres pharmacocinétiques les plus probables au sein d'un ensemble de jeux de paramètres dans la population considérée et calculent la posologie permettant d'atteindre, à moindre coût (au sens général de "prix à payer" en vue d'un but déterminé : "cost-to-go", par exemple l'optimisation de l'ensemble des ressources nécessaires pour atteindre un objectif précis avec un véhicule piloté), la concentration-cible désirée. Pour un horizon donné, ils prennent en compte le fait que les prélèvements sont une contrainte supplémentaire ; de même que les objectifs non réalisés imposent des pénalités, elles-mêmes variables selon les stratégies de commande. Applications de la programmation dynamique stochastique, ils sont à même d'incorporer les diverses sources d'erreur précédemment citées, autres que l'erreur de mesure du feedback (ici l'erreur sur le dosage plasmatique du médicament, l'erreur analytique). Ils sont stochastiques, comme cela a été précédemment défini; ils peuvent alors contrôler de plus larges modèles, éventuellement non linéaires (25).

\subsection{LE RÔLE DU PHARMACOCINÉTICIEN CLINICIEN EN ANTIBIOTHERAPIE}

Le rôle du pharmacocinéticien en antibiothérapie consiste non seulement à doser l'antibiotique mais aussi et surtout au préalable à s'enquérir de la situation clinique, des objectifs du clinicien et à collecter avec soin l'information sur ce qui a déjà été mesuré, déterminé, noté et réellement administré chez le patient, notamment par les infirmières et le laboratoire de microbiologie. Puis il réalise les simulations, propose des schémas posologiques et rend un avis écrit et oral à l'infectiologue en charge du patient.

\subsection{FUTURES TECHNIQUES}

Dans le futur, comme pour d'autres applications du contrôle adaptatif, des techniques dites "closed loop" ne nécessiteront plus d'intervention humaine intermédiaire systématique. Certains contrôleurs de ce type sont déjà utilisés en anesthésiologie.

\subsection{LE ROLE DE LA PHARMACOCINETIQUE DE POPULATION}

Tous les contrôleurs bayésiens utilisant une information a priori nécessitent une description de la répartition des paramètres pharmacocinétiques d'un modèle dans la population correspondant au sujet traité. C'est le rôle des études de pharmacocinétique de population de fournir ces informations.

Les méthodes paramétriques : (1).les méthodes dites en deux étapes : STS, GTS, (2).les méthodes en une étape : NONMEM, P-Pharm, en posant une hypothèse sur la nature de la fonction de densité de probabilité conjointe des paramètres, fournissent les moyennes des paramètres et la matrice de variance-covariance. Leur avantage principal est la simplicité : dans une première étape, sont estimés les paramètres pharmacocinétiques individuels des différents patients constituant la population et dans la seconde, sont calculés les moments caractéristiques d'une distribution gaussienne (moyenne et variance) de ces paramètres. Ces deux premiers moments statistiques sont les seuls utilisés actuellement par les estimateurs dits "Bayésiens". L'inconvénient principal de ces méthodes est qu'une distribution de paramètres 
non gaussienne, par exemple une mixture de gaussienne, imposera de séparer une population initiale en sous-populations qui ne pourront être choisies qu'une par une comme information $a$ priori pour initialiser la thérapeutique avec le contrôleur.

Les méthodes non paramétriques, comme NPML, Popkan ou NPEM, qui ne posent pas d'hypothèses sur la nature de la fonction de distribution des paramètres, peuvent fournir des informations a priori sur des populations très hétérogènes et peuvent alimenter ainsi en information a priori les contrôleurs stochastiques à modèles multiples (35).

\subsection{INTERET DE TELLES METHODES PHARMACOCINETIQUES EN INFECTIOLOGIE}

Ces différentes méthodes ont fait la preuve de leurs capacités à prédire les concentrations sériques en antibiotiques lors d'infections sévères et prolongées ; à ce propos, nous renvoyons le lecteur aux éditions précédentes de cet ouvrage $(32,35)$. Il est par contre beaucoup plus difficile en situation clinique d'évaluer les conséquences de telles méthodes que ce soit en terme d'efficacité finale (guérison) ou encore en termes pharmacoéconomiques, attendu qu'elles permettent l'utilisation de molécules dans des situations impossibles autrement : comment quantifier par exemple le bénéfice d'un traitement par monitoring d'aminosides en gériatrie lors d'une thérapeutique au long cours d'endocardites versus fluoroquinolones à doses standard, toutes choses restant égales par ailleurs ?

\section{PHARMACODYNAMIE DES ANTIBIOTIQUES}

L'activité d'une molécule anti-infectieuse se manifeste par sa capacité à entraîner la disparition du microorganisme responsable de l'infection. Or, il ne s'agit que d'un phénomène populationnel et microscopique qui ne peut être observé directement qu'au laboratoire et non en pratique clinique. En conséquence, comme nous l'avons déjà souligné en introduction, l'efficacité clinique d'une antibiothérapie ne peut être considérée qu'au travers de critères intermédiaires dont les principaux sont cliniques (évolution de l'état clinique du malade, normalisation de la température corporelle), bactériologiques (négativation des prélèvements, titre bactéricide du sérum), biologiques (normalisation des marqueurs de l'infection ou de l'inflammation) et pharmacologiques (atteinte des concentrations plasmatiques dites cibles en terme d'efficacité et de non toxicité) $(23,27)$.

En pratique clinique actuelle, ces critères ne sont donc que rétrospectifs, ce qui rend impossibles leurs utilisations. À l'exception de la détermination a priori des posologies d'antibiotiques par une méthode de pharmacocinétique clinique, comme évoqué précédemment, ces critères ne peuvent être utilisés pour l'optimisation a priori du régime posologique de l'antibiotique, lors de son initialisation. Cependant, avant le début de la thérapeutique, le choix de l'antibiotique et de son régime posologique doit être optimisé afin d'atteindre au plus tôt une efficacité maximale associée à une toxicité minimale. L'intérêt d'une optimisation a priori des posologies est donc double : l'utilisation d'un régime posologique voulu d'emblée efficace doit permettre (i) d'éradiquer les sous-populations bactériennes à sensibilités diminuées (15) et (ii) de réduire les risques de résistance adaptative (10), l'utilisation de doses inadaptées pouvant entraîner une augmentation importante de la CMI du germe (3). 


\subsection{INDICES PHARMACODYNAMIQUES}

En conséquence, une méthode dite d'individualisation duale des posologies a été proposée $(48,47)$. Elle est basée sur l'intégration de données in vitro de bactéricidie (donc, pharmacodynamique) aux données pharmacocinétiques in vivo chez l'homme. Ces données de bactéricidie sont la CMI, voire la CMB. D'autres indices intègrent d'autres types de données de bactéricidie, tels le titre bactéricide du sérum (2) ou les modèles d'effet bactéricide $(30,9)$ plus difficilement accessibles, en routine clinique, que la CMI. Cette intégration repose sur les relations supposées entre le profil des concentrations plasmatiques en antibiotiques et l'activité bactéricide (45) (Figure 1).

Du fait de la quasi-égalité CMI-CMB pour les antibiotiques bactéricides, et du fait que la $\mathrm{CMB}$ ne peut être atteinte en clinique pour les antibiotiques bactériostatiques, son intégration semble peu intéressante. De nombreux indices pharmacodynamiques ont alors été définis pour une période de 24 heures à l'état d'équilibre pharmacocinétique $(15,44,47,50)$; persister à les dénommer "paramètres" maintient, nous semble-t-il, une ambiguïté avec les paramètres pharmacodynamiques tels que nous les avons définis plus haut : ils n'ont, en effet, rien de "dynamique", tous sont calculés à partir de mesures en point final, même si une temporalité est ensuite réintroduite par le calcul. Il nous apparaît, pourtant, indispensable de disposer d'une vision dynamique (dès la première administration) et non plus statique (à l'état d'équilibre pharmacocinétique) de ces indices (7). Leur utilisation doit permettre de juger $a$ posteriori l'efficacité de la thérapeutique antibiotique, mais surtout de prédire a priori cette efficacité et, par conséquent, d'adapter individuellement en terme d'efficacité, le régime posologique d'un antibiotique à un malade donné connaissant la pharmacocinétique de cet antibiotique chez ce malade.

Les indices couramment utilisés sont présentés ci-dessous :

AUC Area Under the plasma antibiotic levels Curve (Aire Sous la Courbe des concentrations plasmatiques en antibiotique, ASC) [mg.h. l $\left.^{-1}\right]$. Elle caractérise, à la fois, la quantité d'antibiotique administrée et la vitesse de son élimination. En d'autres termes, la valeur de l'AUC reflète l'exposition de l'organisme à l'antibiotique.

AUC / MIC AUC weighted by the MIC (ASC rapportée à la CMI) [h]. Cet indice semble corrélé à l'activité des antibiotiques concentration-dépendants tels les fluoroquinolones ou les aminosides $(11,14,49)$.

AUC $>$ MIC AUC mesurée sur les intervalles de temps pendant lesquels les concentrations plasmatiques sont supérieures à la $\mathrm{CMI}$ ou $\mathrm{AUC}$ when $\mathrm{C}>$ MIC [mg.h.l ${ }^{-1}$ ].

AUIC Area Under the Inhibitory Curve (ou Aire Sous la Courbe d'Inhibition ou rapport de ASC > CMI sur la CMI) [h]. Les valeurs cibles de l'AUIC semblent indépendantes de la bactérie et de l'antibiotique. Ainsi, pour les $\beta$-lactamines, les aminoglycosides et les fluoroquinolones, une zone de valeurs pour l'AUIC comprises entre 150 et 250 (voire 350) heures par jour devrait être recherchée $(1,44,49,50)$.

$\mathrm{AUC}_{\mathrm{MIC}} \quad \mathrm{AUC}$ above MIC, souvent notée, à tort $\mathrm{AUC}>\mathrm{MIC}$ définie par $\mathrm{AUC}>\mathrm{MIC}$ déduite de l'aire sous la CMI [mg.h. l $^{-1}$ ]. Chaque antibiotique aurait une valeur cible unique d'AUC $\mathrm{MIC}_{\mathrm{C}}$ pour un germe donné. Cependant, quelle que soit la classe de l'antibiotique, $80 \%$ de l'AUC $_{\text {MIC }}$ maximale (à définir !) 
semble être un seuil minimal à atteindre pour aboutir à une guérison clinique (50).

$\mathrm{T}>\mathrm{MIC}$ Temps [h] ou pourcentage de temps pendant lequel les concentrations plasmatiques sont supérieures à la CMI, soit pour une période de $24 \mathrm{~h}$, soit pour l'intervalle entre les doses. T > MIC est constamment corrélé à l'efficacité thérapeutique, notamment, des $\beta$-lactamines, mais également des autres antibiotiques temps-dépendants (8). Cependant, face à certains germes ( $\mathrm{P}$. aeruginosa), l'activité des antibiotiques concentration-dépendants peut être temps-dépendante (49). L'indice pharmacodynamique optimal est alors $\mathrm{T}>\mathrm{MIC}$.

$\mathrm{C}_{\max }$ / CMI Rapport de la concentration plasmatique maximale sur la CMI ou Quotient Inhibiteur (QI) (17). La valeur de ce rapport a pu être corrélée à l'activité bactéricide in vivo pour les aminoglycosides et les fluoroquinolones $(8,49)$. La valeur optimale (qui peut donc être une valeur cible) de ce rapport serait comprise entre 8 et $10(14,37,38)$. La cinétique du quotient inhibiteur, correspondant au rapport de toutes les concentrations plasmatiques sur la CMI, se rapproche du $\mathrm{T}>\mathrm{CMI}$ mais permet de dire de combien les concentrations plasmatiques sont supérieures à la CMI. Son utilisation est envisageable pour les antibiotiques temps-dépendants, tels les glycopeptides (5).

L'utilisation de ces indices pharmacodynamiques présente un certain nombre d'inconvénients. Tout d'abord, pour un même régime posologique, certains indices ont des valeurs très similaires voire égales. Ces indices doivent également être sensibles aux variations de la valeur de la CMI. Cependant, différents régimes posologiques d'un même antibiotique peuvent conduire à une valeur identique de l'indice considéré $(7,11)$. Ainsi, pour une même dose journalière, la valeur de l'AUC et des indices dérivés (AUC/MIC, AUC $>$ MIC) reste quasiment identique quel que soit le schéma posologique retenu $(1,2$ ou 3 administrations) $(11,21)$. A l'opposé, pour un même rythme d'administration, la valeur de $\mathrm{T}>$ MIC peut rester constante quelle que soit la dose administrée. Puisqu'ils sont multiples, il est difficile de choisir parmi les régimes posologiques, le régime optimal correspondant à la valeur cible de l'indice retenu. Ensuite, T > MIC présente une valeur limite supérieure $(24 \mathrm{~h}$ ou $100 \%$ ) et ne permet donc pas de discriminer deux régimes posologiques efficaces mais différents pour lesquels les valeurs de T $>$ MIC sont de $24 \mathrm{~h}$ ou de $100 \%$ (7). Enfin, ces indices se révèlent spécifiques de la classe de l'antibiotique (Tableau I) et du caractère temps- ou concentration-dépendant de l'activité de l'antibiotique sur la bactérie responsable de l'infection.

Cependant, ces spécificités ne sont pas absolues. Rappelons que, si le rapport $\mathrm{C}_{\max } / \mathrm{MIC}$ est un bon indice de l'activité des aminosides sur la majorité des bactéries gram négatif, $\mathrm{T}>\mathrm{MIC}$ est préférable pour Pseudomonas sp.

En somme, un indice pharmacodynamique idéal devrait être à même de d'indiquer simultanément un certain nombre de caractéristiques dynamiques, auxquelles aucun indice disponible actuellement ne répond de façon satisfaisante, de par sa conception, comme nous l'avons indiqué en introduction de ce paragraphe (Tableau II). 


\subsection{MODÈLES D'EFFETS ET MODÈLES DE BACTÉRICIDIE}

Le terme bactéricide s'applique à l'action de composés chimiques (naturels ou non) ou de procédés susceptibles de tuer les bactéries dans des conditions définies, quel que soit le mécanisme mis en jeu. La mort bactérienne ne peut donc être appréciée de façon individuelle mais seulement à l'échelle de la population bactérienne (12). Seule la diminution du nombre de bactéries viables permet d'objectiver l'activité bactéricide, qui dépend du temps et de la concentration en antibiotique. Ainsi, une certaine relation est attendue entre la concentration d'antibiotique (effecteur), la bactéricidie (effet) et le temps.

L'analyse de l'activité bactéricide des antibiotiques peut être réalisée à concentration constante en antibiotique (in vitro, soit analyse en point final - mesure de la CMI et de la CMB - soit analyse cinétique de l'activité bactéricide ou cinétique de bactéricidie). Les modèles, in vitro ou in vivo chez l'animal, à concentrations fluctuantes en antibiotique permettent de simuler la pharmacocinétique humaine des antibiotiques. Ils se placent d'emblée plus près de la réalité clinique dans laquelle les concentrations en antibiotiques du site infectieux ne sont que très rarement constantes.

Les cinétiques de bactéricidie correspondent à l'analyse in vitro, sur une durée de 18 à 24 heures, de l'activité bactéricide d'un antibiotique à concentration fixe sur un inoculum bactérien de taille connue $\left(10^{+5}-10^{+6} \mathrm{UFC} / \mathrm{ml}\right)$ (Figure 2). L'étude de ces cinétiques montre généralement trois phases distinctes (13). La phase initiale, dite de bactéricidie, est marquée par la diminution (ou l'augmentation) de la taille de la population bactérienne en fonction des valeurs des $\mathrm{CMI}$ et $\mathrm{CMB}$ et de la concentration en antibiotique testée. La deuxième phase est caractérisée par une certaine tolérance de la bactérie vis-à-vis de l'antibiotique ou " résistance adaptative" (équilibre entre croissance et mort bactérienne, même à des concentrations initialement bactéricides). La dernière est dite phase de "recroissance bactérienne". Ainsi, malgré la présence de l'antibiotique à une concentration initialement efficace, la taille de la population bactérienne augmente. Cependant, mise en culture dans un milieu exempt d'antibiotique, cette population bactérienne, dite persistante, présente alors un phénotype de sensibilité et une CMI identiques à ceux d'avant le contact avec l'antibiotique.

L'analyse de la phase initiale de bactéricidie a permis de proposer un modèle pharmacodynamique fonctionnel afin de suivre la réponse d'une population bactérienne exposée à des concentrations fluctuantes en antibiotiques $(54,55)$. Ainsi, la taille, B (UFC/ml), d'une population bactérienne en phase de croissance exponentielle non limitée est fonction de son taux naturel de croissance en absence d'antibiotique, $G\left(h^{-1}\right)$ et de l'effet bactéricide de l'antibiotique, $\mathrm{K}\left(\mathrm{h}^{-1}\right)$.

$$
\frac{d B}{d t}=(G-K) \cdot B
$$

où B est la taille de la population bactérienne (UFC/ml), G son taux naturel de croissance en absence d'antibiotique $\left(\mathrm{h}^{-1}\right)$ et K l'effet bactéricide de l'antibiotique $\left(\mathrm{h}^{-1}\right)$.

Cet effet bactéricide suit un processus non linéaire saturable dépendant du temps, car fonction de la concentration en antibiotique, elle-même fonction du temps :

$$
K_{t}=\frac{K_{\max } \times C_{t}^{\gamma}}{C_{50}^{\gamma}+C_{t}^{\gamma}}
$$

où $\mathrm{K}_{\max }$ est l'effet bactéricide maximal $\left(\mathrm{h}^{-1}\right), \gamma$ le coefficient de sigmoidicité de Hill et $\mathrm{C}_{50}$ la concentration en antibiotique produisant $50 \%$ de $\mathrm{K}_{\max }(\mathrm{mg} / \mathrm{l})$. 
La concentration en antibiotique, pour laquelle le taux apparent de croissance bactérienne, $\mathrm{G}-\mathrm{K}_{\mathrm{t}}$, est nul, correspond à la concentration minimale inhibitrice pharmacodynamique, zCMI (mg/l). En conséquence, la relation entre la zCMI et la $\mathrm{C}_{50}$ est la suivante (36) :

$$
G-\frac{K_{\max } \times{ }_{z} C M I_{t}^{\gamma}}{C_{50}^{\gamma}+z C M I_{t}^{\gamma}}=0 \Leftrightarrow z C M I=C_{50} \cdot \sqrt{\frac{G}{K_{\max }-G}}
$$

Sous ces hypothèses, l'expression $\left(G /\left(K_{\max }-G\right)\right)^{1 / \gamma}$ est une constante et la concentration $\mathrm{C}_{50}$ peut être considérée comme une valeur corrigée ou apparente de la CMI de la souche bactérienne étudiée $(39,42)$. La relation entre zCMI et $\mathrm{C}_{50}$ est donc de type linéaire. En conséquence, plus la valeur de zCMI est élevée, plus celle de $\mathrm{C}_{50}$ l'est également, et plus la moitié de l'effet bactéricide maximal $\left(\mathrm{K}_{\max } / 2\right)$ sera atteinte pour des concentrations élevées d'antibiotiques. Cependant, la nature exacte de la relation entre la CMI et la zCMI reste à définir. Alors que $\mathrm{K}_{\max }$ et $\gamma$ semblent pouvoir définir l'activité bactéricide intrinsèque d'un antibiotique donné face à une espèce bactérienne donnée, la valeur $\mathrm{C}_{50}$ reflèterait la potentialité d'action de cet antibiotique face à une souche donnée de l'espèce bactérienne considérée (28). A titre d'exemple, les valeurs des paramètres, $K_{\max }$ et $\gamma$, sont reproduites pour différents couples bactérie - antibiotique dans le Tableau III.

Selon le type de sigmoïdicité de la fonction de Hill (EQ. 2), les antibiotiques dits concentration-dépendants pour une espèce bactérienne donnée seraient les antibiotiques présentant une assez faible valeur de $\gamma$, ce qui les rendrait actifs dès les basses concentrations (cas de la tobramycine, Tableau III). L'effet maximal ne serait atteint qu'à de fortes concentrations difficilement envisageables en clinique humaine du fait de leur toxicité. A l'inverse, les antibiotiques dits temps-dépendants pour une espèce bactérienne donnée peuvent avoir une assez forte valeur du paramètre $\gamma$ et présentent une toxicité moins importante (cas de l'amoxicilline, Tableau III). La relation existante entre les concentrations en antibiotiques et les effets bactéricides résultants est représentée, pour la tobramycine et pour l'amoxicilline, sur la Figure 3.

L'analyse de ces relations montre l'intérêt d'augmenter les concentrations plasmatiques de tobramycine. En effet, plus les concentrations augmentent, plus l'effet augmente et, cependant, $\mathrm{K}_{\max }$ n'est toujours pas atteint pour de fortes concentrations, par ailleurs non envisageables en clinique. La notion de concentration-dépendance est ainsi confirmée. A l'inverse, le temps de contact entre la souche bactérienne et l'amoxicilline devrait être augmenté, l'effet bactéricide maximal étant rapidement atteint, et cette fois à des concentrations non toxiques. Ainsi, une augmentation des concentrations n'est plus synonyme d'une augmentation de l'effet. L'augmentation de ce temps de présence permet, à lui seul, le maintien de cette activité bactéricide, d'où la notion de tempsdépendance.

Dans la modélisation EQ.1-EQ.2, la repousse bactérienne observée in vitro à concentration constante en antibiotique pour certains couples bactérie - antibiotique (Figure 2) n'est pas prise en compte. In vitro, Reeves et al. (41) ont montré que si la première dose d'antibiotique était efficace, les suivantes pouvaient présenter une efficacité bien inférieure voire nulle. Afin de décrire cette repousse bactérienne, une conversion concentrationdépendante des bactéries d'un état sensible à un état transitoire de persistance (Figure 4) a été intégrée au modèle (29). 
En réponse à la présence de l'antibiotique, le taux de conversion concentrationdépendant, $\delta\left(\mathrm{h}^{-1}\right)$, des bactéries sensibles en bactéries persistantes est donné par :

$$
\begin{aligned}
& \text { si } \mathrm{C}_{\mathrm{t}}<\mathrm{C}_{\mathrm{mec}} \Rightarrow \delta=0 \\
& \operatorname{sic}_{\mathrm{t}} \geq \mathrm{C}_{\mathrm{mec}} \Rightarrow \log _{10} \delta=\xi \cdot\left(\mathrm{C}_{\mathrm{t}}-C_{\text {mec }}\right)+\theta
\end{aligned}
$$

où $\theta$ est le taux intrinsèque de conversion bactérienne de l'état sensible à persistant $\left(\mathrm{h}^{-1}\right)$,

$\xi$ le taux de conversion par unité de concentration $\left(1 . \mathrm{mg}^{-1} \cdot \mathrm{h}^{-1}\right)$

$$
\text { et } \mathrm{C}_{\mathrm{mec}} \text { la concentration déclenchante }(\mathrm{mg} / \mathrm{l}) \text {. }
$$

Ainsi, $\delta$ reste nul tant que la concentration en antibiotique dite déclenchante, $C_{\text {mec }}$ $(\mathrm{mg} / \mathrm{l})$, n'est pas atteinte, y prend alors sa valeur maximale $\left(10^{+\theta} \mathrm{h}^{-1}, \theta<0\right)$ puis décroît selon une exponentielle $(\xi<0)$ alors que la concentration en antibiotique augmente (Figure 5). Au cours d'une administration, la conversion sensible-persistante est effective $(\delta \neq 0)$ au cours de l'augmentation (phase d'administration) et de la diminution (phase d'élimination) des concentrations en antibiotique.

Cependant, les souches bactériennes persistantes peuvent retrouver leur sensibilité initiale $(10,29)$. Ce retour de sensibilité (Schéma 1) n'est effectif (i)qu'en phase d'élimination de l'antibiotique et (ii) que si ces souches sont exposées à des concentrations inférieures à une concentration limite $\left(\mathrm{C}_{0}, \mathrm{mg} / \mathrm{l}\right.$, supposée confondue avec $\left.\mathrm{C}_{\mathrm{mec}}\right)$ pendant une durée minimale $\left(\mathrm{T}_{\mathrm{R}}, \mathrm{h}\right)$, appelé temps de reconversion $(10,29)$. Naudin et al. (40) posent alors comme hypothèse que toutes les bactéries devenues persistantes redeviennent sensibles, phénomène que nous appellerons bascule inverse. Dans ces conditions, l'intervalle entre deux administrations ne peut être que supérieur à ce temps de reconversion. Si ces conditions ne sont pas réunies, les bactéries persistantes échappent, au moins provisoirement, à l'activité de l'antibiotique.

Le couplage d'un modèle pharmacocinétique (PK) avec un modèle pharmacodynamique (PD) présente plusieurs intérêts. Un tel modèle PK-PD peut nous permettre de mieux comprendre les schémas posologiques de certains antibiotiques, utilisés et cliniquement validés en pratique journalière, qui peuvent sembler en contradiction avec les données pharmacocinétiques et pharmacodynamiques actuellement disponibles. Ainsi, $\mathrm{T}>\mathrm{CMI}$, indice corrélé à l'efficacité des $\beta$-lactamines - dont l'amoxicilline -, estimé pour l'amoxicilline administrée deux fois par jour en perfusion intraveineuse de 30 minutes, n'est que de $6.0 \mathrm{~h}(25 \%)$ pour $500 \mathrm{mg}$ et de $7.8 \mathrm{~h} \mathrm{(32.5 \% )} \mathrm{pour} 1000 \mathrm{mg}$ et pour une valeur de CMI fixée à $2 \mathrm{mg} / \mathrm{l}$ (Figure 6). Malgré un temps de contact si court avec la bactérie, l'efficacité thérapeutique de l'amoxicilline a pu être démontrée en clinique. Si nous ne considérons que ce seul indice, l'intervalle entre les doses semble donc trop long. Or pour les $\beta$-lactamines, dès 1984, Gengo et al. (22) envisageaient que l'intervalle entre les doses devait prendre en considération (i) le T > CMI, (ii) un possible effet post-antibiotique et un temps (à définir !) nécessaire à la bactérie pour redevenir sensible à l'antibiotique, ce que reprend le modèle présenté ici et correspond aux travaux d'Eagle datant de 1953 (16).

L'utilisation d'un tel modèle, pharmacocinétique et pharmacodynamique, peut alors être envisagée afin de proposer des schémas posologiques théoriques optimaux, et ce d'un point de vue tout à la fois pharmacocinétique et pharmacodynamique. 


\section{CONDUITE PRATIQUE A TENIR LORS D'UNE ANTIBIOTHERAPIE}

Quelles conséquences pratiques immédiates retenir des deux précédents paragraphes lors d'une antibiothérapie ? Si la première partie, pharmacocinétique, propose des concepts et des outils immédiatement utilisables et validés, la seconde, pharmacodynamique, ne propose pour l'instant que des concepts. Dans ces conditions, comme dans la précédente édition, il s'agit d'optimiser les conditions d'utilisation des outils pharmacocinétiques disponibles au regard des connaissances actuelles de bactéricidie, tout en évitant accumulation et toxicité, essentiellement et encore uniquement dans le cas des traitements par aminoglycosides et glycopeptides (35).

\subsection{Corrélation des concentrations sériques avec la toxicité}

\subsubsection{Aminoglycosides}

Après plus de quarante années d'études de toxicologie expérimentale et d'utilisation clinique simultanée d'au moins deux générations d'aminoglycosides, il est paradoxalement toujours relativement difficile de définir avec précision, en clinique, les conditions d'apparition tant de l'oto- que de la néphro- toxicité, bien que les processus de toxicité en euxmêmes soient assez bien connus. Cette situation résulte de plusieurs facteurs intriqués : la difficulté de transposer les conditions de réalisation des premières études toxicologiques chez l'animal aux conditions cliniques humaines, l'absence de méthodes de dosage suffisamment sensibles et simples pour une généralisation en routine avant 1978, la réalisation fréquente du dosage dans une situation clinique où ne peut jamais être prouvée l'antériorité, soit de l'accumulation, soit de la néphrotoxicité. L'ototoxicité est encore plus difficile à surveiller dans des conditions cliniques de routine. Enfin, il n'est encore que très rarement possible de faire la part entre la variabilité interindividuelle et une situation toxique particulière. Malgré tout il a été démontré que la toxicité peut être réduite de différentes manières souvent associées : par une sélection appropriée des patients, par une limitation des plages "temps" et "dose" des traitements, par un contrôle des facteurs de risque associés avec la toxicité, par l'utilisation de méthodes de modélisation pour élaborer un régime posologique approprié, et enfin par l'usage de schémas posologiques de type "once-a-day". Les deux inconvénients principaux de ces approches empiriques sont, d'une part qu'elles éliminent des sujets "à risques" chez lesquels pourtant un tel traitement serait éminemment justifié et d'autre part qu'elles obligent à un compromis avec - et souvent au détriment de - l'efficacité. Heureusement, les méthodes pharmacocinétiques présentées dans le premier paragraphe permettent de contrôler désormais les concentrations plasmatiques, et à travers elles toxicité et efficacité, pendant de très longues périodes ; un exemple de l'évolution des concentrations sériques et périphériques lors d'un traitement au long cours est représenté sur la Figure 7.

En fait pour les deux types de toxicité, le transport des aminosides dans le tube contourné proximal et dans l'oreille interne apparaît désormais, ainsi que cela a été démontré par des modèles animaux, comme un mécanisme saturable : la toxicité des aminosides est essentiellement liée au temps de contact entre l'antibiotique et les cellules de la bordure en brosse du tube contourné proximal ou les cellules sensorielles cochléovestibulaires. Il apparaît donc logique d'éviter de multiplier le nombre de contacts journaliers de ces tissus avec de fortes concentrations en aminosides, ce qui est en faveur d'un espacement maximum des doses, cependant compatible avec les contraintes de bactéricidie $(19,20)$. 


\subsubsection{Glycopeptides}

Des études rétrospectives réalisées ces quinze dernières années (donc avec des formes pharmaceutiques d'une pureté bien supérieure à celle des premières années de commercialisation) ont évalué la toxicité rénale de la Vancomycine à $5 \%$. Cependant il est encore plus difficile de préciser les circonstances de survenue de la toxicité que dans le cas des aminoglycosides car il n'a pas été mis en évidence de sites d'accumulation de glycopeptides au niveau des tubules rénaux. L'effet sur le rein serait plutôt la conséquence d'une accumulation que le résultat d'une toxicité directe. Des perfusions continues de Vancomycine afin d'utiliser au maximum l'effet temps-dépendant de cette molécule sont désormais réalisées ; dans ces conditions des plateaux inférieurs à $25 \mu \mathrm{g} / \mathrm{ml}$ sont bien tolérés. Le potentiel néphrotoxique de l'association aminoglycosides - Vancomycine serait toujours supérieur à ceux de chaque classe en monothérapie $(17 \%$ à $35 \%$ versus $11 \%$ et $17 \%$ respectivement). Enfin les données concernant la Teicoplanine sont encore plus rares ; une revue générale a cependant relevé respectivement $0.1 \%$ et $0.2 \%$ de néphro- et oto- toxicité chez des patients traités uniquement par la Teicoplanine, ainsi qu'une absence d'effet synergique sur les toxicités de l'association aminoglycosides - Teicoplanine, néanmoins la variabilité de la Teicoplanine est au moins aussi importante que celle de la Vancomycine.

\subsubsection{Fluoroquinolones}

La fréquence des effets indésirables sous fluoroquinolones (Ofloxacine, Péfloxacine, Ciprofloxacine, Sparfloxacine, Trovafloxacine) augmente avec la dose administrée et avec la durée du traitement. Les réactions de photosensibilité sont concentrations plasmatiques dépendantes, notamment des concentrations plasmatiques maximales. Les risques de ruptures tendineuses n'ont pas été reliés à des concentrations plasmatiques excessives (ce qui n'exclue la possibilité d'une concentration-dépendance au niveau de l'articulation) (24).

\subsection{Corrélation des concentrations sériques avec la réponse clinique}

\subsubsection{Aminoglycosides}

\subsubsection{Schéma posologique conventionnel}

Comme nous l'avons vu précédemment, les aminoglycosides sont des antibiotiques dont l'activité bactéricide croît de façon continue avec la concentration, ils sont donc dits essentiellement "concentration-dépendants" ; de plus, ils ont fréquemment un effet postantibiotique marqué (PAE). Moore avait démontré antérieurement qu'un rapport pic / CMI (concentration minimale inhibitrice) supérieur à 4 était associé à environ $70 \%$ de guérison et un rapport supérieur à 10 à $90 \%(37,38)$. Dans ces conditions, bien que la CMI ne soit pas réellement un paramètre pharmacodynamique, pour une bactérie sensible aux aminosides, un objectif satisfaisant, avec des schémas posologiques conventionnels, est un rapport pic / CMI au moins supérieur à 8 (indice appelé précédemment $\mathrm{Cmax} / \mathrm{CMI}$ ).

\subsubsection{Schéma posologique type "once-a-day"}

Les deux concepts précédents de concentration-dépendance et de PAE, ainsi que ceux qui résultent des cinétiques de bactéricidie, comme cela a été développé dans le paragraphe précédent, suggèrent des schémas posologiques produisant des concentrations plasmatiques 
très élevées; d'où le développement du concept de "once-a-day" (20) : une administration unique journalière.

Quoi qu'il en soit, il nous semble qu'avec les outils de contrôle adaptatif désormais à disposition du clinicien, les points importants de cette approche soient (I) l'utilité d'une première dose élevée -massive - (par exemple $20 \mathrm{mg} / \mathrm{kg} / \mathrm{j}$ d'Amikacine, voire $30 \mathrm{mg} / \mathrm{kg} / \mathrm{j}$ chez le neutropénique), (II) la nécessité de prédire et de vérifier les concentrations obtenues par rapport aux objectifs fixés initialement selon la stratégie, et (III) l'obtention périodique de concentrations les plus élevées possibles aux lieux d'action, pendant le temps de traitement nécessaire.

\subsubsection{Glycopeptides}

\subsubsection{Schéma posologique conventionnel}

Les glycopeptides sont dits "temps-dépendants" ; pour une efficacité maximale il importe donc de chercher à augmenter le temps pendant lequel la concentration en antibiotique est supérieure à la PCB (première concentration bactéricide). En pratique, il apparaît qu'une corrélation existe entre les concentrations sériques et le titre bactéricide du sérum : des titres de $1: 2$ à la vallée et de 1:8 au pic semblent augmenter les résultats bactériologiques et cliniques.

\subsubsection{Schéma posologique par perfusions continues}

Certaines équipes ont utilisé pleinement le concept de temps-dépendance pour proposer l'administration de Vancomycine en perfusion continue ; dans ce cas, les concentrations recherchées au plateau sont de 15 à $25 \mu \mathrm{g} / \mathrm{ml}$ selon la sévérité de l'infection. Cependant, il n'est fait état dans ce paragraphe que des relations rapportées entre l'efficacité et les concentrations sériques. Il semble important de rappeler que les titres bactéricides du sérum et ceux du lieu de l'infection peuvent être très différents et d'autre part que les antibiotiques sont sensibles à l'effet inoculum.

En résumé, un suivi thérapeutique lors d'un traitement par les glycopeptides consiste désormais plus à éviter l'accumulation qu'à prévenir la toxicité et à rechercher des concentrations optimales pour un effet temps dépendant.

\subsubsection{Fluoroquinolones}

La relation entre les concentrations plasmatiques des principales fluoroquinolones (Ciprofloxacine, Ofloxacine, Péfloxacine et Trovafloxacine) et l'efficacité clinique a été moins étudiée que dans le cas des aminosides ou des glycopeptides. Les fluoroquinolones sont essentiellement concentration-dépendantes et présentent un effet post-antibiotique modéré non systématique. Les études in vivo chez l'animal ou en clinique humaine montrent en général que l'issue clinique est liée au rapport AUC / MIC ou au quotient inhibiteur pour la majorité des espèces bactériennes $(14,49)$. Cependant, dans certaines situations cliniques, les fluoroquinolones peuvent se révéler temps-dépendantes. En tout état de cause, les concentrations plasmatiques optimales, en terme d'efficacité clinique, des fluoroquinolones restent à préciser dans différentes conditions physiopathologiques pour assurer un bénéfice clinique optimal (24). 


\subsection{Concentrations plasmatiques cibles en situations cliniques courantes}

Enfin, pour répondre aux besoins pratiques immédiats de l'infectiologue, notamment en matière de cibles exprimées sous forme de concentrations plasmatiques, sont reproduits cidessous le tableau (Tableau IV) et les conseils actualisés, qui figuraient en conclusion de la précédente édition (35).

\section{- Lors d'une antibiothérapie prolongée avec aminoglycosides et glycopeptides, un contrôle adaptatif optimal des posologies est nécessaire.}

- Il nécessite la structuration au préalable d'un circuit de recueil des données cliniques et des conditions de la réalisation de la thérapeutique, tout aussi nécessaires que les résultats des dosages plasmatiques d'antibiotique pour l'adaptation de posologie.

- Les prélèvements doivent être effectués non pour constater mais pour prédire, même si les résultats obtenus permettent simultanément une vérification sommaire des prédictions précédentes.

- Les concentrations plasmatiques cibles doivent être envisagées en fonction du germe, de sa localisation, du type de patient, de la gravité de l'infection et des thérapeutiques associées. Par exemple, pour des germes sensibles, chez des adultes jeunes, peuvent être discutées au préalable avec les cliniciens les cibles du Tableau IV. Par analogie et à la vue des résultats publiés (51), figurent également sur ce tableau des cibles possibles pour l'isépamicine. Elles sont données à titre indicatif, pour l'adulte standard ; elles doivent être soigneusement repensées en fonction des situations cliniques individuelles; pour les nouvelles stratégies thérapeutiques des aminosides en particulier, le clinicien doit garder à l'esprit qu'avec les doses administrées, à partir d'une concentration résiduelle non nulle, l'accumulation est très rapide.

- Les simulations permettent de proposer un avis pharmacocinétique écrit dans le dossier médical; le clinicien choisit ensuite les schémas thérapeutiques les plus adaptés au contexte clinique du moment.

\section{CONCLUSIONS}

Les thérapeutiques par médicaments anti-infectieux sont soumises, comme les autres, à la variabilité biologique susceptible de faire différer les réponses d'un patient à un autre. Les techniques de pharmacocinétique clinique appliquées aux antibiotiques permettent d'estimer pour la contrôler, donc d'individualiser les traitements pour une réponse optimale.

Les techniques d'adaptation de posologie modernes ont permis, ces dix dernières années, de réaliser des suivis de traitements antibiotiques, notamment au long cours, en situation clinique courante. Elles ont permis de développer une thérapeutique efficace essentiellement en terme de concentrations sériques obtenues. Elles ont montré, dans un premier temps, les possibilités de l'adaptation de posologie au lit du malade. En générant des dossiers patients plus complets mais correspondant à la situation clinique de tous les jours, elles ont autorisé, dans un second temps, la réalisation d'études de pharmacocinétique de population qui ont enrichi la connaissance des modalités d'action de ces produits. 
Mais pour aller jusqu'à proposer à l'infectiologue un contrôle de l'efficacité de l'antibiothérapie, il est désormais nécessaire de compléter d'abord les modèles pharmacocinétiques par des modèles physiopathologiques permettant d'aller jusqu'au foyer infectieux, puis par des modèles pharmacodynamiques simulant la bactéricidie in vivo.

Le développement de tels modèles pharmacocinétiques / pharmacodynamiques, utilisable en situation clinique, nécessitera, dans un futur proche notamment, d'avoir accès à des méthodes de dosage facile d'emploi (les fabricants de trousses de dosages immunoenzymatiques doivent actualiser leur gamme en fonction non plus de critères de toxicité mais désormais d'efficacité des traitements), de construire et valider des modèles complexes non linéaires, de disposer de méthodes et de facilités de calcul pour estimer les paramètres de tels modèles et proposer un contrôle en temps réel.

En attendant, les indices pharmacodynamiques peuvent être utilisés pour évaluer les chances de réussite d'une antibiothérapie, notamment lorsque leur usage est couplé avec celui des logiciels d'adaptation de posologie existants $(7,36)$.

\section{GLOSSAIRE DE NOTIONS PROBABILISTES UTILISEES DANS LES PARAGRAPHES 1.3 A 1.8}

- Fonctions de densité, conjointe, marginale.

La fonction de densité $\mathrm{f}(\mathrm{x})$ d'une grandeur ou variable aléatoire $\mathrm{X}$ continue (i.e. d'un paramètre pharmacocinétique) associe, à chaque valeur que peut prendre la variable considérée, la densité de probabilité de réalisation de cette valeur par la variable aléatoire.

En d'autre terme, la probabilité que la variable $\mathrm{X}$ prenne sa valeur dans l'intervalle $[\mathrm{x}$, $\mathrm{x}+\mathrm{dx}[\operatorname{vaut} \mathrm{f}(\mathrm{x}) \mathrm{dx}$.

Dans le cas d'un couple de grandeurs aléatoires (X, Y), par exemple un couple composé d'une quantité injectée et d'une constante d'élimination, ou vecteur aléatoire, on peut définir, de manière similaire, une densité de probabilité conjointe $\mathrm{f}(\mathrm{x}, \mathrm{y})$ correspondant à la densité de probabilité de réalisation du couple de valeurs $(\mathrm{x}, \mathrm{y})$. Ainsi, la probabilité que le couple $(X, Y)$ prenne ses valeurs dans le pavé de coordonnées de sommets $[\mathrm{x}, \mathrm{y}] ; \mathrm{x}+\mathrm{dx} ; \mathrm{y}+\mathrm{dy}]$ vaut $\mathrm{f}(\mathrm{x}, \mathrm{y}) \mathrm{dxdy}$.

On désigne par fonction de densité marginale la fonction de densité correspondant à une des composantes $\mathrm{X}$ ou $\mathrm{Y}$ du couple $(\mathrm{X}, \mathrm{Y})$, quelle que soit la valeur prise par l'autre composante. On a $\mathrm{fX}(\mathrm{y})=\operatorname{somme}(\mathrm{f}(\mathrm{x}, \mathrm{y}) \mathrm{dy})$.

\section{- Fonctions de densité a priori, posteriori.}

La fonction de densité conjointe d'une ou d'un couple de variables aléatoires continues avant que l'événement associé ne se soit produit (aucune valeur disponible de concentration plasmatique en médicament) est dite a priori.

La fonction de densité conjointe d'une ou d'un couple de variables aléatoires continues actualisée en utilisant le théorème de Bayes après que l'événement associé se soit produit (des mesures de concentration sanguine en médicament ont été réalisées) est alors dite a posteriori.

\section{- Probabilité conditionnelle.}

La probabilité de réalisation de l'événement $\mathrm{A}$ sachant que l'évènement $\mathrm{B}$ s'est réalisé est appelée la probabilité conditionnelle $\mathrm{P}(\mathrm{A} / \mathrm{B})$. Cette valeur est égale à la probabilité de l'évènement conjoint $(A, B)$ divisé par la probabilité de l'évènement $B$. 
Le théorème de Bayes permet d'inverser cette formulation en l'exprimant en fonction de l'évènement $\mathrm{P}(\mathrm{B} / \mathrm{A})$, inversant en quelque sorte le sens d'une relation de causation et le point de vue: ainsi si un évènement $\mathrm{E}$ est susceptible d'avoir été "causé" par une série de causes $\mathrm{Ci}$ possibles selon une certaine distribution a priori, $\mathrm{p}(\mathrm{E} / \mathrm{Ci})$, il est possible d'estimer la "cause" la plus probable sachant que l'évènement $\mathrm{E}$ s'est produit :

$\mathrm{P}(\mathrm{Ci} / \mathrm{E})=\mathrm{P}(\mathrm{E} / \mathrm{Ci}) \mathrm{P}(\mathrm{Ci}) /$ somme $\mathrm{P}(\mathrm{E} / \mathrm{Ck}) \mathrm{P}(\mathrm{Ck})$.

Exemple: clairance de l'aminoside - paramètre pharmacocinétique - cause a, clairance de la créatinine - covariable - cause b et concentration plasmatique mesurée - événement réalisé.

\section{- Estimation, estimateur.}

Les paramètres définissant un modèle pharmacocinétique prennent, pour chaque individu, des valeurs différentes, définissant ainsi sur l'ensemble des individus une grandeur aléatoire caractérisée par la connaissance de sa densité de probabilité et de quelques paramètres résumant sa distribution: la moyenne représente un tel résumé. Ses valeurs diffèrent en fonction des individus étudiés. Pour chaque échantillon d'individus, il est possible d'exhiber soit un critère dépendant des paramètres inconnus du modèle comparant les valeurs mesurées chez les individus et les valeurs telles que prédites par le modèle (critère des moindres carrés), soit une probabilité de réalisation ou vraisemblance des données effectivement observées également dépendante des paramètres inconnus du modèle, qui sera maximisée selon un point de vue résolument pragmatique et réaliste (critère du maximum de vraisemblance).

Un estimateur statistique est une variable aléatoire, dont une réalisation se calcule à partir des données observées.

\section{- Optimisation.}

C'est l'opération consistant à trouver la meilleure solution à un problème, (i.e. les valeurs les plus probables des paramètres pharmacocinétiques d'un patient ou d'une population). Elle peut être sujette à des contraintes.

Des techniques d'optimisation sont utilisées dans des procédures statistiques comme les moindres carrés et le maximum de vraisemblance.

A côté des méthodes classiques sont désormais utilisées des méthodes de programmation mathématique: programmation linéaire, programmation non linéaire, programmation dynamique.

\section{- Contrôleur, zone thérapeutique.}

Dans un système contrôlé par régulation active avec rétrocontrôle interviennent en fait trois sous-systèmes :

- un sous-système opérateur qui subit les entrées et produit les sorties du système (i.e. les doses administrées, les concentrations plasmatiques, l'organisme du patient),

- un sous-système régulateur / estimateur qui recueille les informations (mesures), puis estime ou optimise, en utilisant les mesures, les probabilités des valeurs des paramètres du système (i.e. la clairance de l'aminoside administré),

- et enfin un sous-système contrôleur qui définit les finalités du système sous forme de normes qui regroupent tout à la fois les objectifs et les contraintes (i.e. la zone thérapeutique, c'est-à-dire l'étendue des concentrations sanguines en médicament admissibles et/ou leurs valeurs minimales ou maximales : pics, résiduels, ... à l'intérieur de cette zone thérapeutique). 


\section{REFERENCES BIBLIOGRAPHIQUES}

1. Amsden, G.W., C.H. Ballow, and J.J. Schentag. 1993. Population pharmacokinetic methods to optimize antibiotic effects. Drug. Invest. 5: 256-268.

2. Barriere, S.L., E. Ely, J.E. Kapusnik, and J.G. Gambertoglio. 1985. Analysis of a new method for assessing activity of combinations of antimicrobials: area under the bacterial activity curve. J. Antimicrob. Chemother. 16: 49-59.

3. Blaser, J., B.S. Stone, M.C. Groner, and S.H. Zinner. 1987. Comparative study with enoxacine and netilmicin in a pharmacodynamic model to determine importance of ratio of antibiotic peak concentration to MIC for bactericidal activity and emergence of resistance. Antimicrob. Agents Chemother. 3: 1054-1060.

4. Bouvier d'Yvoire, M.J.Y., and P.H. Maire. 1996. Dosage regimen of antimicrobials. Implications of a pharmacokinetic/pharmacodynamic model. Clin. Drug. Invest. 11: 229239.

5. Cohen, R., E. Varon, H. Dabernat, J. Barre, a,d P. Geslin. 1991. Activité antibactérienne et pharmacocinétique sérique des $\beta$-lactamines orales vis à vis de Streptococcus pneumoniae et de Haemophilus influenzae. La lettre de l'infectiologue. 4: 416-419.

6. Confesson M.A., X. Barbaut, P. Maire, J.M. Vergnaud, A. El Brouzi, and R.W. Jelliffe. 1994. Concentrations calculées en aminoside dans des végétations d'endocardites - relation avec les pratiques cliniques lors du traitement d'endocardites infectieuses par l'amikacine. Thérap.49: 27-34.

7. Corvaisier, S., P.H. Maire, M.Y. Bouvier d'Yvoire, X. Barbaut, N. Bleyzac, and R.W. Jelliffe. 1998. Comparison between antimicrobial pharmacodynamic indices and bacterial killing as described by using the Zhi model. Antimicrob. Agents Chemother. 42: 17311737.

8. Craig, W.A. 1998. Pharmacokinetic / pharmacodynamic parameters : rationale for antibacterial dosing of mice and men. Clin. Infect. Dis. 26:1-12.

9. Czock, D., and M. Giehl. 1995. Aminoglycoside pharmacokinetics and -dynamics: a nonlinear approach. Intern. J. Clin. Pharmacol. Therap. 33: 537-539.

10. Daïkos, G.L., G.G. Jackson, V.T. Lolans, and D.M. Livemore. 1990. Adaptive resistance to aminoglycoside antibiotics from first-exposure down-regulation. J. Infect. Dis. 162: 414-420.

11. Dalla Costa, T., and H. Derendorf. 1996. AUIC - A general target for the optimization of dosing regimens of antibiotics? Ann. Pharmacother. 30: 1024-1028.

12. Drugeon, H., F. Legallou F, and J. Caillon. 1990. Méthodes d'étude de l'activité bactéricide, p. 113-126. In P. Courvalin, H. Drugeon, J.P. Flandrois, and F. Goldstein (ed.) Bactéricidie : Aspects théoriques et thérapeutiques-1990. Maloine, Paris.

13. Drugeon, H.B. 1993. L'amikacine administrée en dose unique journalière : les arguments bactériologiques. Med. Mal. Infect. 23: 5-13.

14. Dudley, M.N. 1991. Pharmacodynamics and pharmacokinetics of antibiotics with special reference to the fluoroquinolones. Am. J. Med. 91(Suppl. 6A): 45-50. 
15. Dudley, M.N. 1992. Commentary on dual individualization with antibiotics, p. 18.118.13. In W.E. Evans, J.J. Schentag, and W.J. Jusko (ed.) Applied pharmacokinetics : Principles of therapeutic drug monitoring, $3^{\text {rd }}$ ed-1992. Applied Therapeutics Inc, Vancouver.

16. Eagle H., R. Fleischman, and M. Levy. 1953. "Continuous" vs. "discontinuous" therapy with penicillin : the effect of the interval between injections on therapeutic efficacy. $\mathrm{N}$. Engl. J. Med. 248: 481-488.

17. Ellner, P.D., and H.C. Neu. 1981. The inhibitory quotient. JAMA. 246: 1575-1578.

18. Evans W.E., Relling M.V., Rodman J.H., Crom W.R., Boyett J.M., Pui C.H. 1998. Conventional compared with individualized chemotherapy for childhood acute lymphoblastic leukemia. N. Engl. J. Med. 338: 499-505.

19. Fantin, B., and C. Carbon. 1993. Arguments en faveur de l'administration en dose unique journalière des aminosides : données expérimentales. Med. Mal. Infect. 23: 14-21.

20. Ferriols-Lisart, R., and M. Alós-Almiñana. 1996. Effectiveness and safety of once-daily aminoglycosides : a meta-analysis. Am. J. Health-Syst. Pharm. 53: 1141-1150.

21. Flückiger, U. C. Segessenmann, and A.U. Gerber. 1991. Integration of pharmacodynamic of imipenem in human-adapted mouse model. Antimicrob. Agents Chemother. 35: 1905-1910.

22. Gengo, F.M., T.W. Mannion, C.H. Nightingale, and J.J. Schentag. 1984. Integration of pharmacokinetics and pharmacodynamics of methicillin in curative treatment of experimental endocarditis. J. Antimicrob. Chemother. 10: 619-631.

23. Grillot, M., P.H. Maire, M.A. Confesson, V. Cogoluenhes, C. Pivot, M. Druguet, P. Courpron, and R.W. Jelliffe. 1994. Contrôle adaptatif des thérapeutiques par l'amikacine chez des patients très agés : analyse rétrospective de l'efficacité et de la toxicité. Path. Biol. 42: 247-253.

24. Hooper, D.C., and J.C. Wolfson. 1993. Quinolone antimicrobial agents, $2^{\text {nd }}$ ed-1993. American society for microbiology Inc, Washington, D.C. 549 p.

25. Jelliffe R.W., A. Schumitzky, D. Bayard, M. Milman, M. van Guilder, X. Wang, F. Jiang, X. Barbaut, and P. Maire. 1998. Model-based, goal-oriented, individualised drug therapy - linkage of population modelling, new "multiple model" dosage design, bayesian feedback and individualised target goals. Clin. Pharmacokinet. 34: 57-77.

26. Jelliffe, R.W., A. Schumitzky, M. van Guilder, M. Liu, L. Hu, P. Maire, P. Gomis, X. Barbaut, and B. Tahani. 1993. Individualizing drug dosage regimens : roles of population pharmacokinetic and dynamic models, Bayesian fitting, and adaptive control. Ther. Drug Monit. 15: 380-393.

27. Kashuba, A.D.M., A.N. Nafziger, G.L. Drusano, and J.S. Bertino. 1998. Dosing of aminoglycosides to rapidly attain pharmacodynamic goals and hasten therapeutic response by using individualized pharmacokinetic monitoring of patients with pneumonia caused by gram-negative organisms.Antimicrob. Agents Chemother. 1998. 42: 1842-1844.

28. Li, R.C. 1996. Simultaneous pharmacodynamic analysis of the lag and bactericidal phases exhibited by $\beta$-lactams against Escherichia coli. Antimicrob. Agents Chemother. 40 : 2306-2310. 
29. Li, R.C., D.E. Nix, and J.J. Schentag. 1994. Pharmacodynamic modeling of bacterial kinetics : $\beta$-lactams antibiotics against Escherichia coli. J. Pharm. Sci. 83 :970-975.

30. Madaras-Kelly, K.J., B.E. Ostergaard, L. Baeker-Hovde, and J.C. Rotschafer. 1996. Twenty-four-hour area under the concentration-time curve/MIC ratio as a generic predictor of fluoroquinolone antimicrobial effect by using three strains of Pseudomonas aeruginosa and an in vitro pharmacodynamic model. Antimicrob Agent Chemather. 40 : 627-632.

31. Maire P., X. Barbaut, J.M. Vergnaud, M. El Brouzi, M.A. Confesson, C. Pivot, M. Chuzeville, N. Ivanoff, J.L. Brazier, and R.W. Jelliffe. 1994. Computation of drug concentrations in endocardial vegetations in patients during antibiotic therapy. Int. J. Biomed. Comput. 36 : 77-85.

32. Maire, P.H., J.L. Brazier, and R.W. Jelliffe. 1992. Pharmacocinétique appliquée aux antibiotiques, p. 251-277. In : J. Freney, F. Renaud, W. Hansen, C. Bollet (ed) Manuel de Bactériologie Clinique, $1^{\text {st }}$ ed, t.I-1992. Elsevier, Paris.

33. Maire, P.H., N. Bleyzac, R.W. Jelliffe, and J.C. Thalabard. 1995. Théorie de l'adaptation de posologie chez le sujet âgé. Act. Pharm. Biol. Clin. 8 : 171-182.

34. Maire, P.H., R.W. Jelliffe, C. Dumarest, D. Roux, V. Bréant, B. Charpiat, E. Vermeulen, J.L. Brazier, and P. Courpron. 1989. Contrôle adaptatif optimal des posologies: expérience des aminosides en gériatrie, p. 154-169. In : A. Venot, P. Degoulet (ed) Informatique et Médicaments-1989. Springer-Verlag, Paris.

35. Maire, P.H., X. Barbaut, J.C. Thalabard, F. Mentré, and R.W. Jelliffe. 1994. Pharmacocinétique appliquée aux antibiotiques, p. 479-518. In : J. Freney, F. Renaud, W. Hansen, C. Bollet (ed) Manuel de Bactériologie Clinique, $2^{\text {nd }}$ ed, t.I-1994. Elsevier, Paris.

36. Maire, P.H., X. Barbaut, J.C. Thalabard, J.M. Vergnaud, D. Roux, M. Roy, and R.W. Jelliffe. 1995. Adaptive control of therapeutic drug regimens relations between clinical situations : outcomes and simulations using nonlinear dynamic model, p. 11111115. In R.A. Greenes, H.E. Peterson, and D.J. Protti (ed.) Proceedings of the $8^{\text {th }}$ World Congress on Medical Informatics-1995. International Medical Informatics Association, Edmonton.

37. Moore, R.D., C.R. Smith, and P.S. Lietman. 1984. Association of aminoglycoside plasma levels with therapeutic outcome in a gram-negative pneumonia. Am. J. Med. 77 : 657-662.

38. Moore, R.D., P.S. Lietman, and C.R. Smith. 1987. Clinical response to aminoglycosides therapy: importance of the ratio of peak concentration to minimal inhibitory concentration. J. Infect. Dis. 155 : 93-99.

39. Mouton, J.W., A.A.T.M.M. Vinks, and N.P. Punt. 1997. Pharmacokineticpharmacodynamic modeling of activity of ceftazidime during continuous and intermittent infusion. Antimicrob. Agents Chemother. 41 : 733-738.

40. Naudin, J., A. Kamenka, D. Claude, and P. Maire. 1997. Automatique en biologie : Etude pharmacocinétique / pharmacodynamique d'un modèle de persistance bactérienne. Données non publiées.

41. Reeves, D.S. 1985. Avantages and disavantages of an in vitro model with two compartments connected by a dialyser : results of experiments with ciprofloxacin. J. Antimicrob. Chemother. 15(Suppl. A) : 159-167. 
42. Renard, L., P. Sanders, and M. Laurentie. 1993. Modélisation de l'effet bactéricide de la spiramycine. Choix d'un modèle pharmacodynamique. Vet. Res. 24 : 33-45.

43. Renard, L., P. sanders, M. Laurentie, and J.M. Delmas. 1996. Pharmacokineticpharmacodynamic model for spiramycin in staphylococcal mastitis. J. Vet. Pharmacol. Therap. 19 : 95-103.

44. Rotschafer, J.C., K.J. Walker, K.J. Kelly, and C.J. Sullivan. 1994. Antibiotic pharmacodynamics, p. 315-343. In N.R. Culter, J.J. Sramek, and P.K. Narang (ed.) Pharmacodynamics and drug development : perspectives in clinical pharmacology-1994. John Wiley \& Sons Ltd, Chichester.

45. Rotschafer, J.C., R.A. Zabinski, and K.A. Walker. 1992. Pharmacodynamic factors of antibiotic efficacy. Pharmacother. 12 : 64S-70S.

46. Rowland, M., L.B. Sheiner, and J.L. Steimer. 1985. Variability in drug therapy : description, estimation, and control - a Sandoz workshop. Raven Press - New York. 246 p.

47. Schentag, J.J., C.H. Ballow, Paladino J.A., and D.E. Nix. 1992. Dual individualization with antibiotics : integrated antibiotic. Management strategies for use in hospitals, p. 17.1-17.20. In W.E. Evans, J.J. Schentag, and W.J. Jusko (ed.) Applied pharmacokinetics : Principles of therapeutic drug monitoring, $3^{\text {rd }}$ ed-1992. Applied Therapeutics Inc, Vancouver.

48. Schentag, J.J., D. Swanson, and I. Smith. 1985. Dual individualization : antibiotic dosage calculation fom the integration of in-vitrp pharmacodynamics and in-vivo pharmacokinetics. J. Antimicrob. Chemother. 15(Suppl. A) : 47-57.

49. Schentag, J.J., D.E. Nix E., and A. Forrest. 1993. Pharmacodynamics of the fluoroquinolones, p. 259-271. In D.C. Hooper, and J.S. Wolfson (ed.) Quinolone antimicrobial agents, $2^{\text {nd }}$ ed-1993. American society for microbiology Inc, Washington, D.C.

50. Schentag, J.J., D.E. Nix, and M.H. Adelman. 1991. Mathematical examination of dual individualization principles : relationships between AUC above MIC and area under the inhibitory curve for cefmenoxime, ciprofloxacin and tobramycin. DICP Ann. Pharmacother. 25 : 1050-1057.

51. Singlas, E. 1996. Pharmacocinétique clinique de l'isépamicine. La lettre de l'infectiologue. Numéro Hors Série, 26-30.

52. Staneva, M., B. Markova, I. Atanasova, and D. Terziivanov. 1994. Pharmacokinetic and pharmacodynamic approach for comparing two therapeutic regimens using amikacin. Antimicrob. Agents Chemother. 38 : 981-985.

53. Vance-Bryan, K., T.A. Larson, J.C. Rotschafer, and J.P. Toscano. 1992. Investigation of the early killing of Staphylococcus aureus by daptomycin by using a in vitro pharmacodynamic model. Antimicrob. Agents Chemother. 36 : 2334-2337.

54. Zhi, J., C.H. Nightingale, and R. Quintilini. 1986. A pharmacodynamic model for the activity of antibiotics against microorganisms under nonsaturable conditions. J. Pharm. Sci. 75 : 1063-1067.

55. Zhi, J., C.H. Nightingale, and R. Quintilini. 1988. Microbial pharmacodynamics of piperacillin in neutropenic mice of systemic infection due to Pseudomonas aeruginosa. J. Pharmacokin. Biopharm. 16 : 355-375. 
Tableau I : Indices pharmacodynamiques corrélés à l'efficacité antibactérienne.

\begin{tabular}{ll}
\hline Indices & Classe d'antibiotique \\
\hline$T>$ MIC & Pénicilline, céphalosporine, carbapenème, macrolide, lincosamide \\
AUC/MIC sur $24 h$ & Aminoside, fluoroquinolone, tétracycline, glycopeptide, synergystine \\
$C_{\max } /$ MIC & Aminoside, fluoroquinolone \\
\hline
\end{tabular}

Tableau II : Caractéristiques de AUC, AUC>MIC, T>MIC, AUC/MIC et $C_{\max } / M I C$.

\begin{tabular}{|c|c|c|c|c|c|}
\hline Caractéristiques & $A U C$ & $A U C>M I C$ & $T>M I C$ & $A U C / M I C$ & $C_{\max } / M I C$ \\
\hline Détermination aisée & $+{ }^{*}$ & $-{ }^{* *}$ & - & + & ++ \\
\hline Incorporation de tous les paramètres $P K$ & + & + & $+/-$ & + & - \\
\hline Incorporation de la CMI & - & + & + & + & + \\
\hline Sensibilité aux changements de CMI & - & - & $+/-$ & + & + \\
\hline Vision dynamique & + & + & + & + & - \\
\hline Absence de discontinuité & + & - & - & + & - \\
\hline Absence de valeur maximale & + & + & - & + & + \\
\hline Antibiotique concentration-dépendant & + & + & - & + & + \\
\hline Antibiotique temps-dépendant & - & - & + & - & - \\
\hline Relation claire avec la bactéricidie & - & - & $+/-$ & $+/-$ & $+/-$ \\
\hline Analyse des associations d'antibiotiques & - & - & - & - & - \\
\hline
\end{tabular}

* présence, ${ }^{* *}$ absence de la caractéristique mentionnée

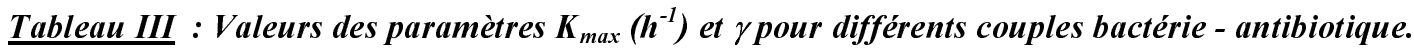

\begin{tabular}{ccccc}
\hline Antibiotique & Espèce bactérienne & $K_{\max }\left(h^{-1}\right)$ & $\gamma$ & Références \\
\hline Amoxicilline & Escherichia coli & 4.160 & 5.470 & 23 \\
Amikacine & Pseudomonas aeruginosa & 6.580 & $1.000 *$ & 52 \\
Ticarcilline & $"$ & 1.882 & 0.902 & 4 \\
Tobramycine & $"$ & 7.115 & 0.416 & 4 \\
Daptomycine & Staphylococcus aureus & 7.640 & $1.000 *$ & 53 \\
Spiramycine & $"$ & 1.924 & 1.279 & 43 \\
\hline
\end{tabular}

* valeur arbitrairement fixée égale à 1 


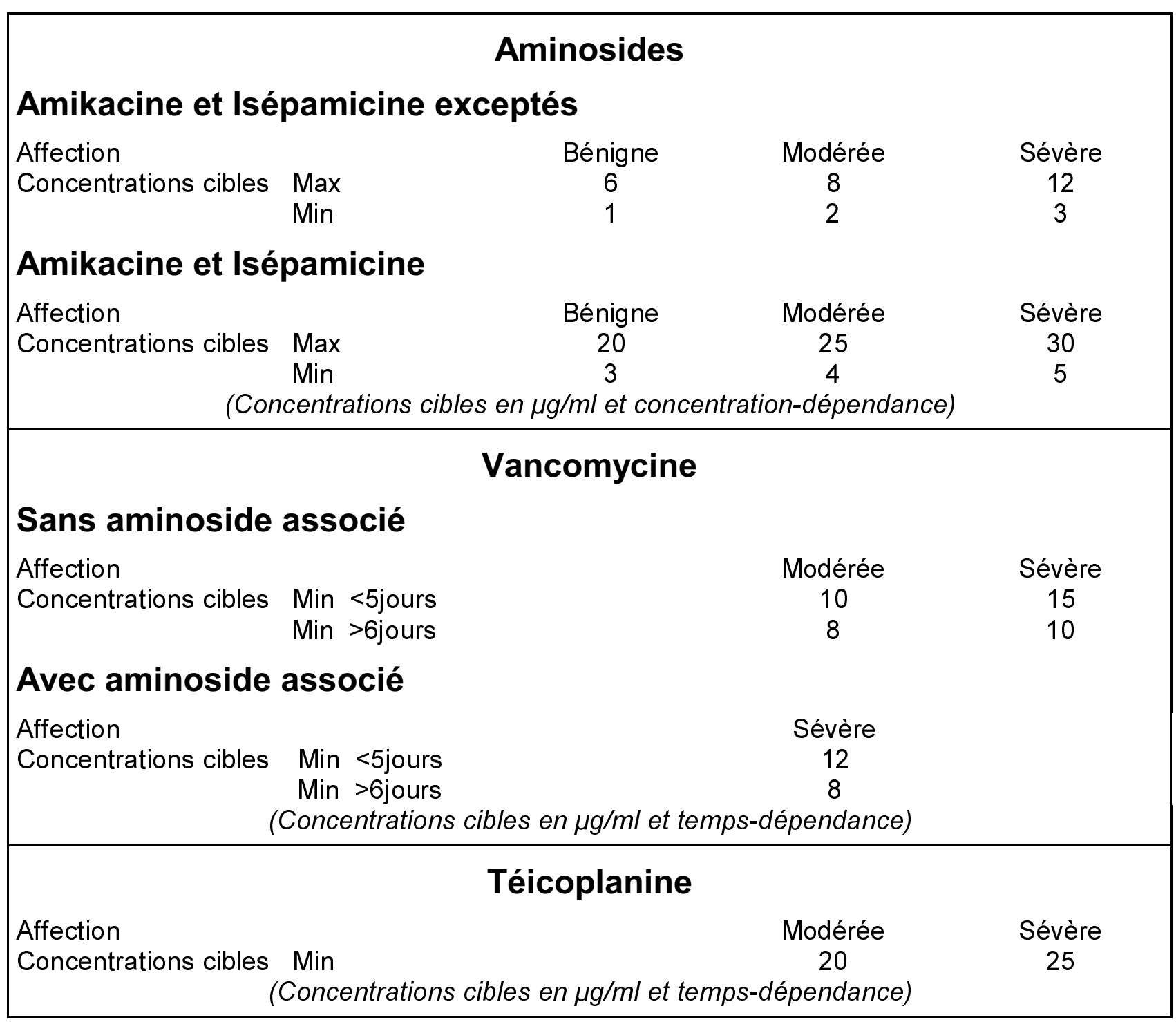




\section{Aminosides - Stratégie "Once-A-Day"}

\section{Amikacine et Isépamicine exceptés}

Affection

Concentrations cibles Max

Min

Bénigne
12
0

Modérée
14
0

Sévère

$>16$

$<1$

Amikacine et Isépamicine

Affection

Concentrations cibles Max

Bénigne

40

Modérée

50

Sévère

Min

0

0

(Concentrations cibles en $\mu \mathrm{g} / \mathrm{ml}$ et concentration-dépendance)

\section{Vancomycine (sans aminoside associé)} Perfusion Continue

Affection

Modérée

15

Sévère

Concentrations cibles Plateau

(Concentrations cibles en $\mu \mathrm{g} / \mathrm{m} /$ et temps-dépendance)

Ces cibles, données à titre indicatif, sont une synthèse d'expériences cliniques et des Données Actuelles de la Science. En aucun cas, elles ne sauraient engager la responsabilité des auteurs. 


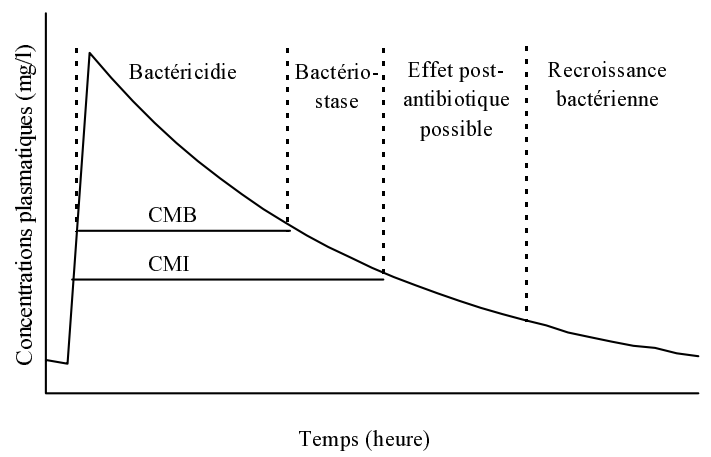

Figure 1 : Relations entre les concentrations plasmatiques et l'activité anti-bactérienne.

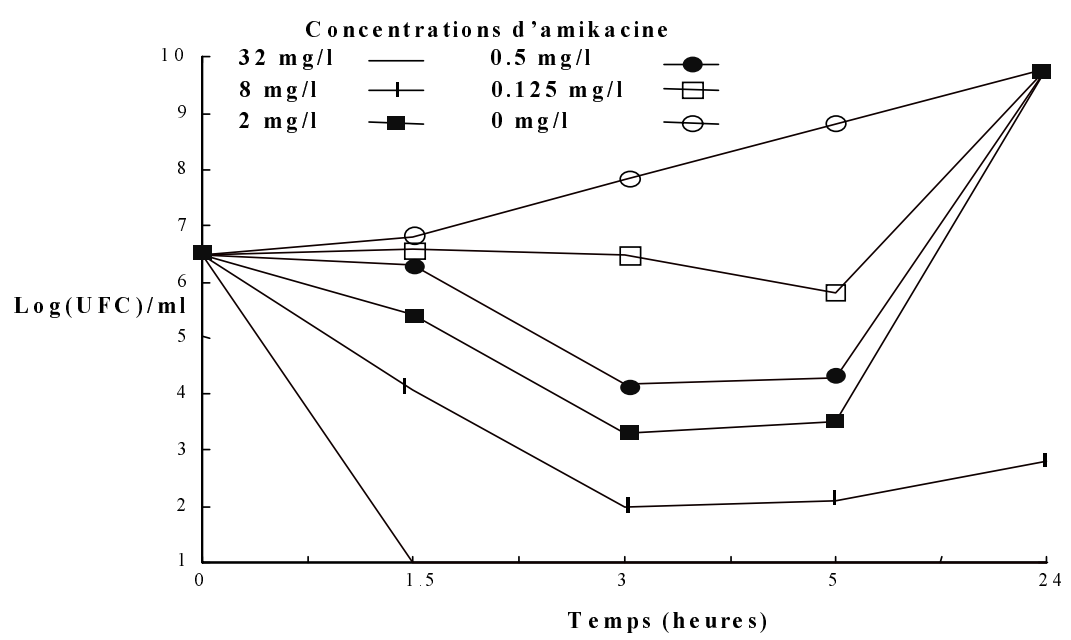

Figure 2 : Cinétique de bactéricidie de l'amikacine face à P. aeruginosa.

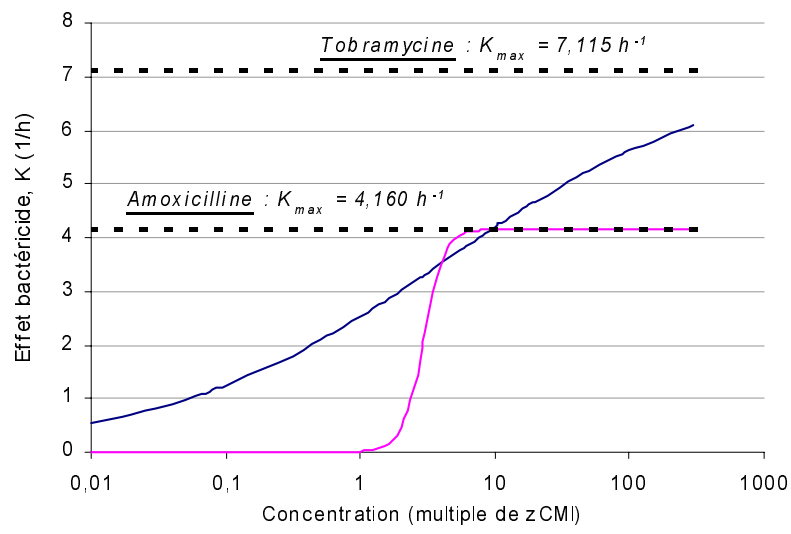

Figure 3 : Effet bactéricide, $K\left(h^{-1}\right)$, de la tobramycine sur P. aeruginosa ATCC 27853 et de l'amoxicilline sur E. coli ATCC 25922.

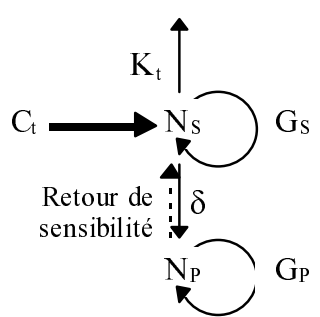

où $C_{t}$ est la concentration en antibiotique ( $\left.\mathrm{mg} / \mathrm{l}\right)$ à l'instant $t(h)$, $N_{S}$ et $N_{P}$ les tailles des populations bactériennes respectivement sensibles et persistantes (UFC/ml), $G_{S}$ et $G_{P}$ les taux de croissance en absence d'antibiotique des populations respectivement sensibles et persistantes $\left(h^{-1}\right)$ (supposés confondus) et $\delta$ le taux de conversion des bactéries sensibles en persistantes $\left(h^{-1}\right)$.

Figure 4 : Conversion bactérienne concentration-dépendante d'un état sensible à un état persistant (23). 


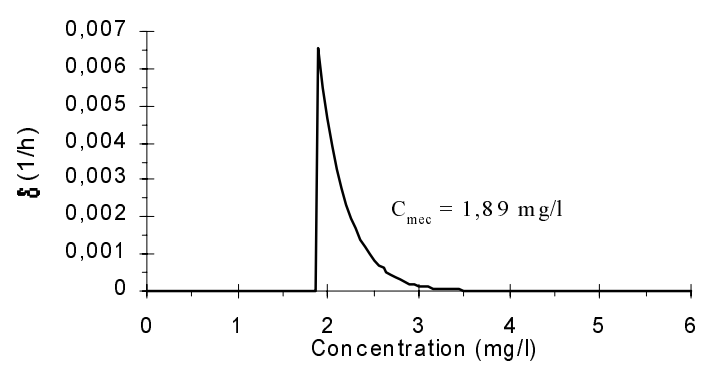

$\underline{\text { Figure } 5}$ : Evolution de $\delta\left(h^{-1}\right)$ de E. coli fonction de la concentration en amoxicilline.

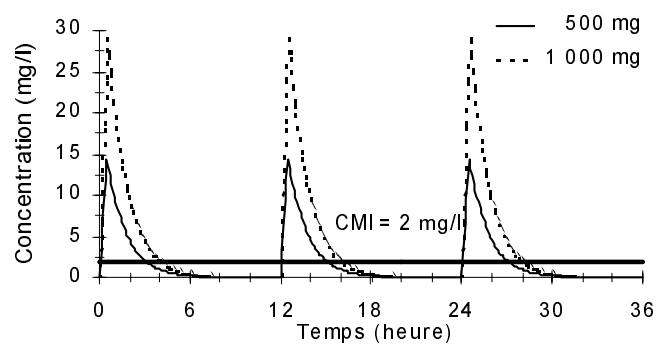

Figure 6: Concentrations plasmatiques (mg/l) de l'amoxicilline (perfusion IV de 30 min).

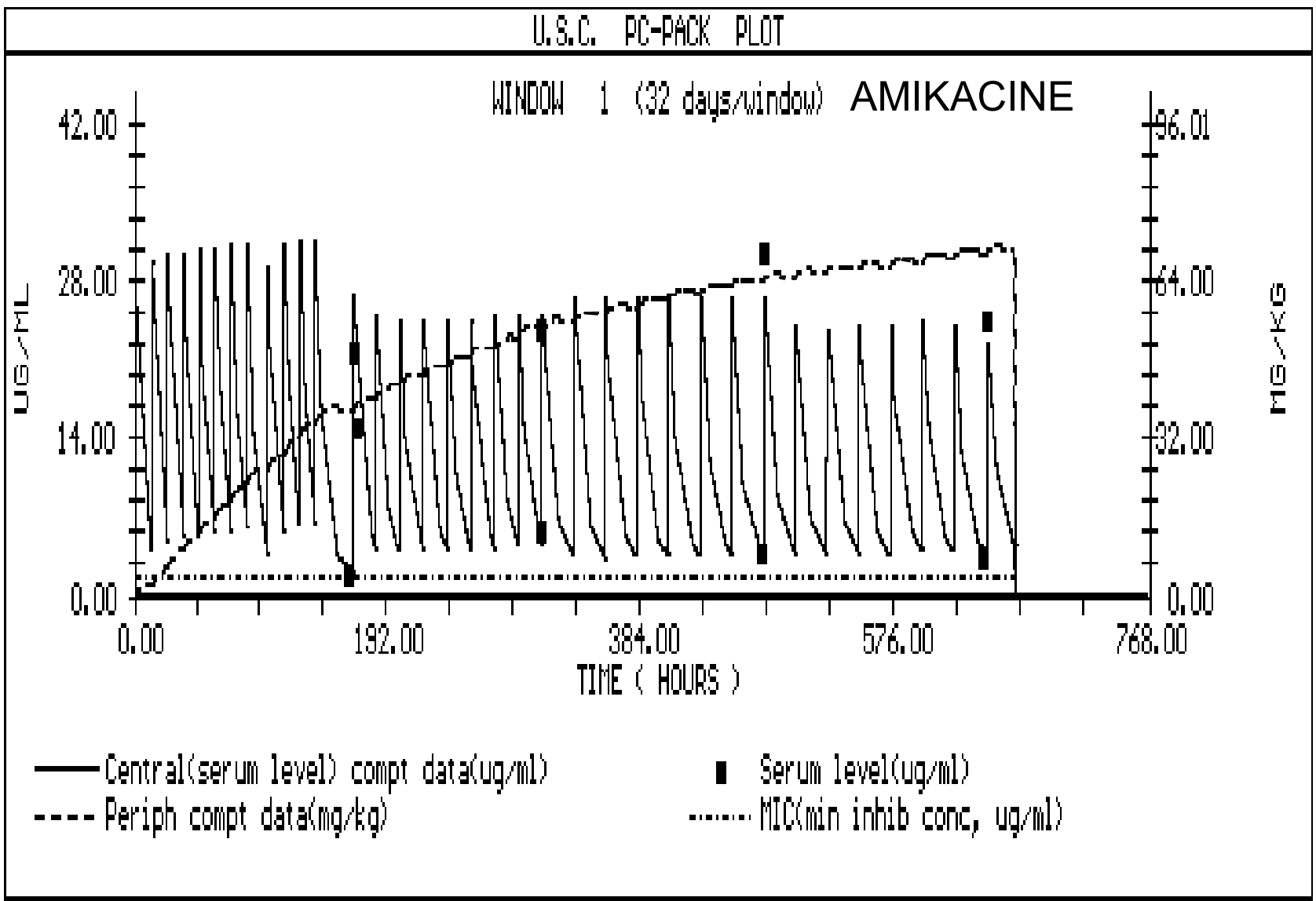

Figure 7: Une situation clinique typique. Le contrôle adaptatif a assuré des maxima efficaces en aminoside tout en évitant l'accumulation. 